\title{
AN INVESTIGATION OF THE SOURCES OF EARTH-DIRECTED SOLAR WIND DURING CARRINGTON ROTATION 2053
}

\author{
A. N. FAZAKerley ${ }^{1}$, L. K. Harra ${ }^{1}$, and L. van Driel-Gesztelyi ${ }^{1,2,3}$ \\ ${ }^{1}$ Mullard Space Science Laboratory, University College London, UK; a.fazakerley@ucl.ac.uk \\ ${ }^{2}$ Konkoly Observatory of the Hungarian Academy of Sciences, Budapest, Hungary \\ ${ }^{3}$ Observatoire de Paris, LESIA, FRE 2461(CNRS), F-92195 Meudon Principal Cedex, France \\ Received 2014 December 17; accepted 2016 April 2; published 2016 June 1
}

\begin{abstract}
In this work we analyze multiple sources of solar wind through a full Carrington Rotation (CR 2053) by analyzing the solar data through spectroscopic observations of the plasma upflow regions and the in situ data of the wind itself. Following earlier authors, we link solar and in situ observations by a combination of ballistic backmapping and potential-field source-surface modeling. We find three sources of fast solar wind that are low-latitude coronal holes. The coronal holes do not produce a steady fast wind, but rather a wind with rapid fluctuations. The coronal spectroscopic data from Hinode's Extreme Ultraviolet Imaging Spectrometer show a mixture of upflow and downflow regions highlighting the complexity of the coronal hole, with the upflows being dominant. There is a mix of open and multi-scale closed magnetic fields in this region whose (interchange) reconnections are consistent with the up- and downflows they generate being viewed through an optically thin corona, and with the strahl directions and freeze-in temperatures found in in situ data. At the boundary of slow and fast wind streams there are three short periods of enhanced-velocity solar wind, which we term intermediate based on their in situ characteristics. These are related to active regions that are located beside coronal holes. The active regions have different magnetic configurations, from bipolar through tripolar to quadrupolar, and we discuss the mechanisms to produce this intermediate wind, and the important role that the open field of coronal holes adjacent to closed-field active regions plays in the process.
\end{abstract}

Key words: magnetic reconnection - solar wind - Sun: abundances - Sun: corona

\section{INTRODUCTION}

The earliest model of solar wind (Parker 1958) proposed the existence of a radial outflow of solar wind from a spherically symmetric solar surface along radial (open) magnetic field lines. In reality, solar remote sensing data show that closed magnetic loops cover a large part of the solar surface, and in situ spacecraft observations in the heliosphere show that the properties of the solar wind are not spherically symmetric or stationary in time.

The speed distribution of the solar wind is continuous, but a distinction is often made between a "fast" and a "slow" solar wind, based on differences not only in speed but also in density, composition (heavy ion charge states), and distribution in heliocentric latitude (which shows a variation with solar cycle). Low latitudes are usually dominated by slow wind and high latitudes by fast wind except near solar maximum.

Solar wind plasma can only travel into the heliosphere on open magnetic flux or highly extended loops. To assess how solar surface magnetic field may reach into the heliosphere, a potential-field source-surface model (PFSS, introduced by Schatten et al. 1969) is typically used with boundary conditions from solar magnetograms. A radial potential field is imposed at a selected distance from the Sun, commonly 2.5 solar radii. Luhmann et al. (2002) used the PFSS technique to assess the locations of open magnetic flux over three solar cycles and found that at solar minimum most open flux comes from the polar regions while at solar maximum most open flux comes from mid and low latitudes.

Gloeckler et al. (2003) examined data from most of a solar cycle (10 years) and showed that the bulk speed of the solar wind is anticorrelated with the coronal electron temperature (inferred from ratios of ion charge states in the solar wind), except in the special case of coronal mass ejections. The relationship is well preserved even for solar wind material whose other plasma characteristics have been modified significantly since leaving the Sun, thus ratios of ion charge states in the solar wind are a key tool for linking coronal and solar wind observations. The temperatures were interpreted as an indicator of heights of magnetic loops in the coronal regions from which the measured solar wind plasma originates, on the assumption that plasma on these loops is released into the solar wind following magnetic reconnection with open flux. Gloeckler et al. presented evidence from Ulysses covering a range of helio-latitudes, and in particular compared solar wind originating in polar coronal holes (predominantly open flux) and that from the quiet Sun (predominantly closed flux). In this and other studies, typical inferred coronal electron temperatures for sources of fast wind from polar coronal hole are $\sim 1.1 \mathrm{MK}$, while inferred coronal electron temperatures for slow wind associated with the streamer belt were found to be more variable in the range 1.4-2.0 MK. Based on this study and similar work, the fast solar wind is now widely considered to originate in (polar) coronal holes.

Spectroscopic studies of coronal holes have concentrated on jets and bright points within the coronal holes, the coronal hole boundaries, and differences with bright points in the quiet Sun (e.g., Kamio et al. 2011; Madjarska et al. 2012), and on searches for evidence of waves propagating from the coronal holes into the fast solar wind (e.g., Banerjee et al. 2009; Bemporad \& Abbo 2012). Coronal hole boundaries where open and closed magnetic fields coexist are known to provide multiple jet sources. High-density plasma has been measured from these, indicating a potential source of the slow solar wind. Although the bright points in the quiet Sun are also associated 
with jets, their plasma flows remain confined in closed loops (Narang et al. 2016).

Wang et al. (2009) studied ACE data for the interval 1998-2007 and proposed that, as well as fast wind, two varieties of slow wind can be distinguished using ratios of ion charge states. They argue that slow wind originating inside the polar coronal hole boundaries and in low-latitude coronal holes has slightly higher $\mathrm{O}^{7+} / \mathrm{O}^{6+}$ ratios than wind from the centers of polar coronal holes (median value $0.14, T_{\mathrm{e}} \sim 1.05 \mathrm{MK}$ for declining phase and solar minimum, 2003-2007) while slow wind associated with active regions has higher ratios (median value $0.25, T_{\mathrm{e}} \sim 1.5 \mathrm{MK}$ for solar maximum 1999-2002). The authors present a model that examines how the speed and composition of outflowing plasma on flux tubes may be affected by the magnetic field strength of the flux tube footprint, the degree of heating at the coronal base of the flux tube, the degree of heating at higher altitudes, and the degree to which the flux tube expands with increasing altitude above the coronal base. Different scenarios were used in three model runs to show how low, intermediate and high charge state ratios might arise in the observed relationship to wind speed.

Liewer et al. (2004) studied specific cases (from the data set used in a statistical study by Neugebauer et al. 2002) of solar wind near solar maximum, which appeared to have open-field solar wind sources associated with active regions. In their studies, an active region was defined as a region of strong magnetic field with no corresponding coronal hole in $\mathrm{He}$ $10830 \AA$ maps from Kitt Peak NSO. The examples of "active region wind" typically had oxygen freeze-in temperatures $\sim 1.6-1.9 \mathrm{MK}$. This is somewhat cooler than typical temperatures on loops deep within active regions $(>2 \mathrm{MK})$, and the authors suggest that the sources may have been long, high cooler loops, similar to those observed near solar maximum connecting different active regions with temperatures 1-1.4 MK (Feldman et al. 1999). It was suggested that the existence of a range of temperatures reflected a range of loop sizes within this class of loop. The coronal hole wind at solar maximum had a higher coronal temperature than at solar minimum, $<1.6 \mathrm{MK}$ compared to $<1.2 \mathrm{MK}$ for polar coronal holes at solar minimum. A set of events where the source could not be definitively located as either coronal hole or active region showed temperatures $\sim 1.6-1.7 \mathrm{MK}$. In the majority of cases studied, the open flux predicted by PFSS models mapped to the edge of an active region, usually where a darker feature was visible in EUV or soft X-ray images. In one case, a doubledipole coronal magnetic structure was found in the PFSS model, with open flux originating at the separatrix between loops connecting regions of opposite polarity well inside the active region. A key result of this paper and others referred to therein is that solar wind does escape on open flux in or near active regions, so that traditional "coronal holes" defined in terms of regions of low emissivity on soft X-ray and/or EUV images are not the exclusive locations of open magnetic flux.

Schrijver \& DeRosa (2003) used a flux dispersal model and a Solar and Heliospheric Observatory (SOHO) MDI data assimilation procedure together with the PFSS technique to investigate source regions of solar wind. In two case studies, Schrijver and DeRosa showed that open flux associated with active regions is typically long-lived and associated with one side of the active region. This result is consistent with the findings of Liewer et al. (2004) and with the results of a study by Kojima et al. (1999) that suggested that slow solar wind does not originate from the opening of closed magnetic loops above an active region, but rather from the rapidly expanding (with increasing altitude) flux associated with one polarity side of the active region.

Observations during solar minimum made by the Hinode satellite show the presence of persistent hot plasma upflows at the edges of many active regions. Sakao et al. (2007) reported continuous upflow of plasma from the edge of active region AR 10942, observed in X-rays with the Hinode X-ray Telescope (XRT) and with the EUV Imaging Spectrometer (EIS). The upflow source had an estimated temperature of 1.1 MK. The flow persisted for at least three days. The upflow speeds were around $50 \mathrm{~km} \mathrm{~s}^{-1}$; however, the line profiles are known to sometimes show faint blue-wing asymmetries with speeds of over $100 \mathrm{~km} \mathrm{~s}^{-1}$ (Hara et al. 2008). Sakao et al. argued that a PFSS study showed that open flux from the upflow region was open into the heliosphere and that if all the observed upflowing plasma escaped, it could contribute up to $25 \%$ of the total mass loss rate of the solar wind. In a recent work Brooks et al. (2015) carried out detailed full-Sun mapping of the properties of upflowing plasma and a comparison with open magnetic field paths. They showed that active region upflows can supply $50 \%-80 \%$ of slow-wind plasma observed at ACE. These upflow regions exist at a coronal hole/active region boundary, over monopolar magnetic field (Doschek et al. 2008) similar to examples cited above. The flows are fastest in hotter coronal lines (Del Zanna 2008).

Studies have been carried out to understand the location and generation mechanism(s) of the strong upflows. Baker et al. (2009) demonstrated that the outflowing regions lie at locations where magnetic field lines display strong gradients in magnetic connectivity, i.e., at "quasi-separatrix layer" (QSL) surfaces or separatrices, where electric currents accumulate and magnetic reconnection can take place, driving the flows through the creation of a pressure gradient. Scott et al. (2013) confirmed in various magnetic configurations and on a wide range of scales that plasma upflows originate at the boundary of different magnetic connectivities. Quasi-separatrices include, as extremes, separatrices linked to null points. Del Zanna et al. (2011) and van Driel-Gesztelyi et al. (2012) found that magnetic (interchange) reconnection at high-altitude null points in pseudo-streamer configuration is responsible for channeling upflow plasma from active regions into the solar wind.

Brooks \& Warren (2012) measured the first ionization potential (FIP) bias of these plasma upflow regions and found it to be between 3 and 5, similar to characteristic FIP values in slow solar wind, which implies that (at least some of) these plasma upflows may contribute to the slow solar wind. Since it takes time for the plasma to evolve to coronal composition this indicates that the plasma within these strong flows has probably been trapped on coronal loops before escaping. Their findings are more difficult to understand in the framework of a model with chromospheric origin, such as the proposal by McIntosh \& De Pontieu (2009) that the upflows observed are consistent with a chromospheric origin associated with spicules.

The linkage between the upflows and the solar wind has been discussed many times. However, it is challenging to make a definitive connection between the upflows observed in remote sensing data that lie low in the corona and the large-scale structures that form the solar wind in the heliosphere. The spatial scales are different, and the physical processes occurring in the different regimes will be different. Ko et al. (2006) 
analyzed data collected over a period of a week during which there was an equatorial coronal hole and an active region using $\mathrm{SOHO/UVCS,} \mathrm{which} \mathrm{observed} \mathrm{at} 1.64$ solar radii. By using an abundance analysis with UVCS data and correlating this with in situ abundance measurements, they confirmed that some slow solar wind did indeed come from the boundary between the coronal hole and active region. Work has been carried out to correlate the fan loops so frequently seen in active regions with the large-scale coronal rays seen above the limb out to 2.5 solar radii by Slemzin et al. (2013). A strong correlation was found between these structures, which were related to an active region and a nearby coronal hole, and open field lines indicated in a PFSS model. The structures were further tracked by studying the in situ data, which confirmed the identification of the active region/coronal hole complex as a source region of the slow solar wind. Spectroscopic measurements of the activeregion flows in the high corona (between 1.5 and 2.3 solar radii) were carried out by Zangrilli \& Poletto (2012). They measured outflow speeds and densities, and these turned out to be confined to a narrow channel at the edge of closed loop systems within the active region. They also found that the outflow speeds from these active regions, measured at heliocentric distances between 1.5 and 2.3 solar radii were faster than streams from equatorial coronal holes.

Modeling is becoming increasingly accurate in determining locations of open field lines and locations where reconnection is favorable. Van Driel-Gesztelyi et al. (2012) used a combination of detailed magnetic field extrapolations at the level of active regions and global potential-field source-surface modeling combined with both remote sensing and in situ data to determine whether or not some of the plasma outflows from the periphery of an active region would find their way to the solar wind, and confirmed that active-region plasma is indeed released into the solar wind via interchange reconnection at a high-altitude null point. Culhane et al. (2014) and Mandrini et al. (2014) could even show that this is possible via a multiple-step reconnection process from below streamers. These results were also consistent with the idea that active regions bordering on coronal holes or linked to a null point in the vicinity of a coronal hole boundary can contribute to the slow solar wind. In all the active regions there were upflows measured low down, but not all of these can escape, and some remain confined. Understanding the surroundings and global topology allows greater insight into the creation of solar wind.

The aim of this paper is to investigate the solar sources of Earth-directed solar wind, as reported from spacecraft in the vicinity of the Earth-Sun L1 point. It is well established that coronal holes are the source of fast solar wind, but there are several models of sources of slow solar wind (e.g., summarized in Antiochos et al. 2011). To explore the sources of both fast and slow solar wind, we choose to study Carrington Rotation 2053, from 2007 February 04 20:04 UT to March 04 04:09 UT, which occurred just before the recent solar minimum. The interval is of interest since although there were several active regions on the Sun, no interplanetary coronal mass ejections (ICMEs) were observed at L1, reducing the complexity of the situation compared to more active times. The active regions were all close to the solar equator, some adjacent to coronal holes, while others were far from open field, allowing us to analyze and discuss the sources of both intermediate-speed and fast wind and test whether the wind observations could be linked with plasma upflows in active regions.
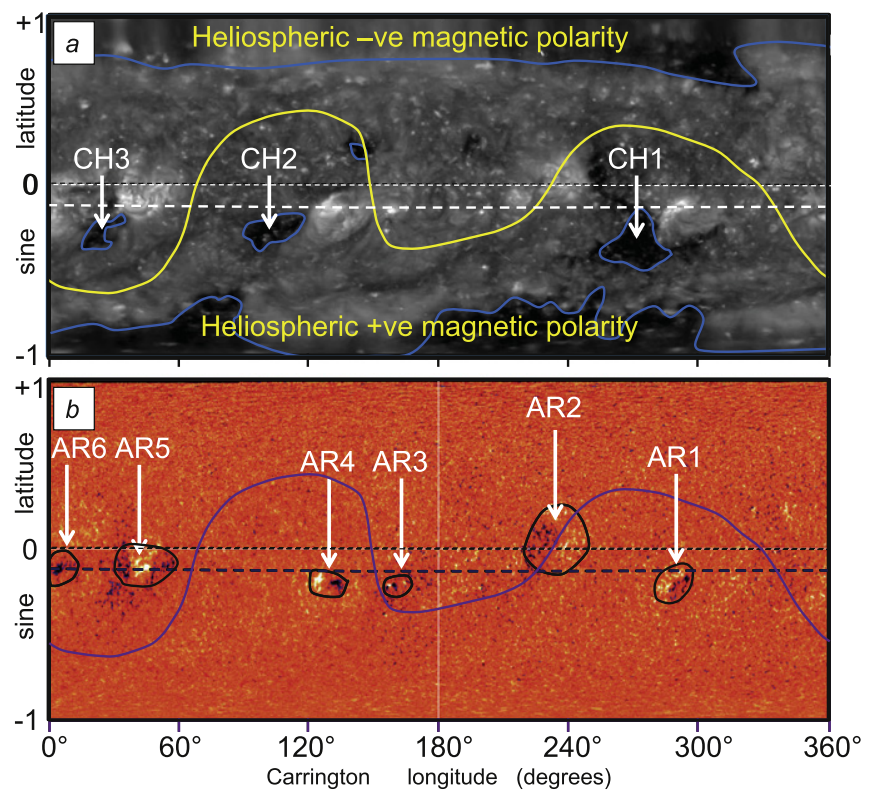

Figure 1. Overview of solar data for CR 2053 (time runs from right to left while Carrington longitude increases from left to right). Panel (a) shows EUV emission data provided by STEREO-EUVI, showing coronal holes and active regions. Approximate coronal hole boundaries have been added in blue. Panel (b) is a synoptic map from $\mathrm{SOHO}$-MDI, showing areas of intense magnetic fields and their polarity, highlighting the active regions whose approximate boundaries have been added in black. The dotted line shows the solar equator while the dashed line shows the footprint latitude of spacecraft at L1. The solid curve shows the footprint of the heliospheric current sheet according to a WSO model that assumes a radial field at a $2.5 R_{\mathrm{S}}$ source-surface, separating regions of positive (away from the Sun) and negative (toward the Sun) heliospheric magnetic field. Solar east is to the left, west to the right.

\section{OVERVIEW OF CARRINGTON ROTATION 2053}

\subsection{Solar Observations}

Figure 1 shows synoptic charts of magnetogram data from SOHO MDI and of EUV data from STEREO EUVI. The observations used to build up the Carrington synoptic maps are created by taking a section of the solar image each day. Time therefore runs from right to left. The horizontal dotted lines represent $0^{\circ}$ heliographic latitude. The dashed horizontal lines represent the latitude beneath the $A C E$ spacecraft during this interval, at about $-7^{\circ}$ latitude; radial outflow from here would reach a spacecraft at $\mathrm{L} 1$. The solid curve represents the approximate footprint of the heliospheric current sheet (HCS) as determined by the Wilcox Solar Observatory (WSO) model of the coronal source-surface magnetic field, computed assuming a radial field at $2.5 R_{\mathrm{s}}$, which separates predominantly negative (inward directed) magnetic field in the northern hemisphere from predominantly positive (outward directed) magnetic field in the southern hemisphere. Three nearequatorial coronal holes are labeled on the EUV plot, all of which lie close to the L1 footprint. Six active regions are labeled on the MDI magnetograms, which show areas of intense magnetic field, typically associated with active regions, and their polarity. The EUV data show the hot, dense plasma associated with energy release in these active regions. All the active regions lie close to the track of the $\mathrm{L} 1$ footprint.

A coronal hole/active region pair is found at a Carrington longitude of $\sim 290^{\circ}$ (CH1/AR1). A second such pair is found at $\sim 120^{\circ}$ (CH2/AR4). The latter case is the coronal hole/active region studied by Sakao et al. (2007), Harra et al. (2008), Baker 
et al. (2009), and He et al. (2010b). In both cases, the active region lies to the west of the coronal hole, and the magnetic field of the coronal hole has the same positive polarity as the adjacent magnetic flux from the neighboring active region. The interaction of AR3 and AR4 makes this a quadrupolar configuration. A third coronal hole/active region pair is seen at $30^{\circ}$ (CH3/AR5) but in this case the active region is tripolar. The magnetic polarity of the coronal hole is negative and again it lies adjacent to magnetic flux of the same polarity from an active region.

An isolated bipolar active region is seen at $\sim 230^{\circ}$ (AR2), which lies a little further from the L1 footprint track than the others. A bipolar active region is seen at $\sim 160^{\circ}$ (AR3), which is a weaker near-neighbor of AR4. Harra et al. (2008) proposed, supported by magnetic extrapolations, that AR3 and AR4 are connected by overlying magnetic flux loops. Finally, at $\sim 5^{\circ}$ there is the remnant of a strong negativepolarity magnetic region (AR6) that generated ICMEs in 2006 December, which is thought to have excess negative magnetic flux and an anemone-like magnetic field configuration, with open flux in the central region.

\subsection{In Situ Plasma Observations at L1}

The interval of interplanetary data at L1 corresponding to solar Carrington Rotation 2053 starts and finishes later than the solar interval due to the transit time of solar wind material from the Sun to L1. The solar wind speed varies from 250 to $700 \mathrm{~km} \mathrm{~s}^{-1}$ during this period. Simple ballistic backmapping (e.g., Nolte \& Roelof 1973) assumes that the flow speed of the solar wind measured at L1 has not changed since that wind material left the Sun and considers how much the Sun has rotated while that material travelled from the Sun to L1. Using this approach, we estimate that the relevant interval at L1 is within the time span 2007 February 08 14:00 UT to March 07 05:00 UT.

Figure 2 provides an overview of the properties of the solar wind and interplanetary magnetic field (IMF) observed in the vicinity of L1 during the selected time interval. The passage of the sector boundaries in the heliospheric magnetic field is revealed by reversals in the dominant pitch angle of the strahl electron distributions of the solar wind (Crooker et al. 2004). The blue lines in Figure 2(b) are proxies for the dominant strahl pitch angle, positive for $0^{\circ}$ and negative for $180^{\circ}$, and show sector boundary crossings on February 12, 22, and 25, and March 04. The strahl is always directed anti-sunward, but the pitch angle will reverse if the polarity of the IMF at the strahl source has reversed. Intervals where the direction of the radial component of the local IMF is opposite to that expected according to the strahl direction indicate that the magnetic field has been bent back on itself, which may indicate interchange reconnection in the solar wind (Crooker et al. 2004).

The bulk speed of the solar wind shows three multi-day intervals of "fast" wind (speed $>450 \mathrm{~km} \mathrm{~s}^{-1}$ ) separated by slower wind, and the density and temperature of the solar wind, together with the IMF intensity, show the expected enhancements in the vicinity of the fast-slow stream interaction regions. The fast wind is associated with low freeze-in temperatures (inferred from charge state ratios of carbon and oxygen) as expected for wind of coronal hole origin, while the slowest solar wind is associated with the highest freeze-in temperatures.

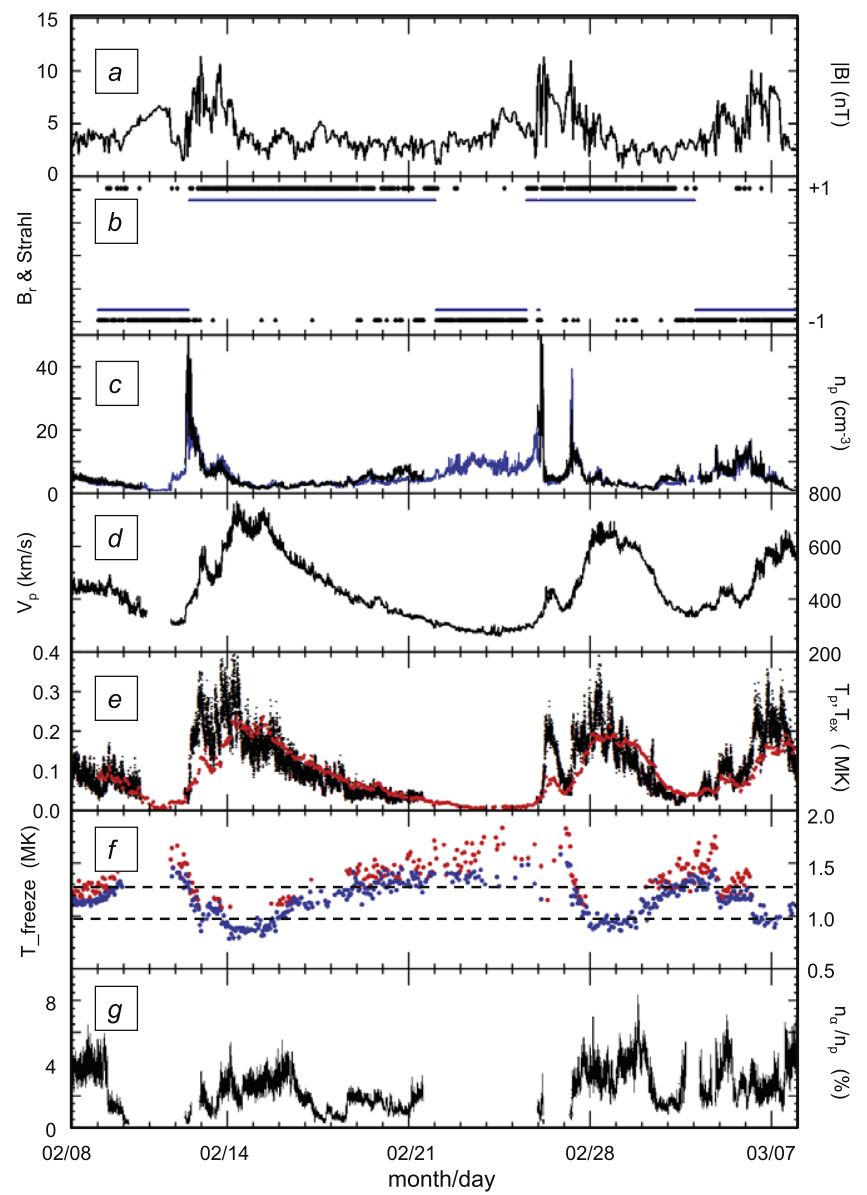

Figure 2. Overview of interplanetary data from 2007 February 08 to March 08. Panel (a) shows the magnetic field intensity. Panel (b) shows whether the interplanetary magnetic field (black) is directed toward (negative) or away from the Sun (positive), and shows the dominant strahl pitch angle (blue), positive for $0^{\circ}$ and negative for $180^{\circ}$. Panel (c) shows the proton density in the solar wind from ACE (black) and WIND (blue). Panel (d) shows the bulk flow speed of the solar wind. Panel (e) shows the proton temperature of the solar wind in black, together with the "expected temperature" in red derived from solar wind speed. Panel (f) shows "freeze-in" temperatures inferred from the ion charge state ratios of oxygen (red) and carbon (blue). Panel (g) shows the ratio of the alpha and proton densities in the solar wind. Data are from $A C E$ unless otherwise indicated.

During this time interval, we found no evidence of ICMEs, using typical search criteria (Zurbuchen \& Richardson 2006). For example, as seen in Figure 2 there are no intervals with alpha/proton density ratio $\left(n_{\alpha} / n_{\mathrm{p}}\right)$ above $8 \%$ or proton density below $1 \mathrm{~cm}^{-3}$, and proton temperature does not fall well below the "expected" temperature for the observed solar wind speed (Richardson \& Cane 1995). We did not see evidence of a magnetic cloud such as steadily rotating, minimally fluctuating magnetic field, nor did we see a prolonged interval of bidirectional strahl electrons.

\subsection{Relating in Situ Data from L1 to Solar Observations}

Figure 3 shows the backmapped solar wind data together with a PFSS synoptic plot, with the boundaries of coronal holes and active regions, and the modeled HCS location from Figure 1 superimposed. The dashed white lines represent positive (away from the Sun) open magnetic flux and the solid white lines represent negative (toward the Sun) open magnetic flux. The magnetic flux is plotted from the photosphere up to a 


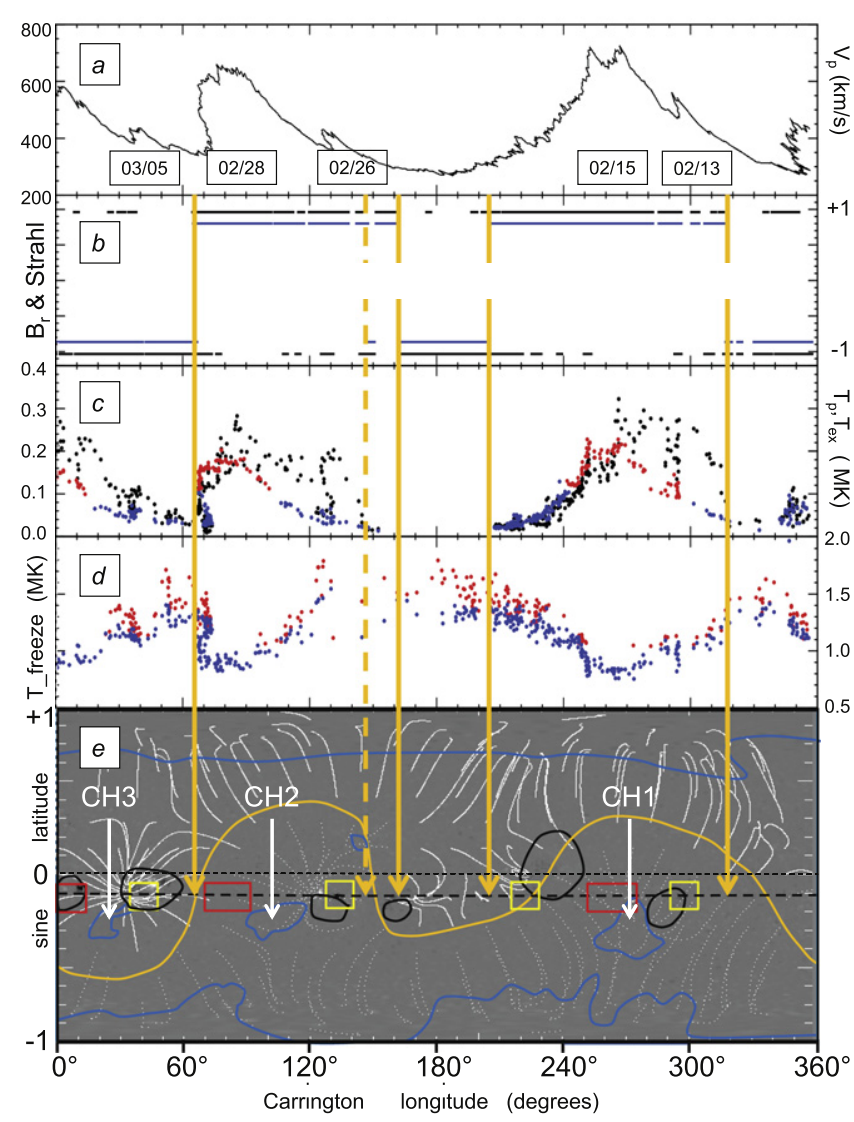

Figure 3. Overview of ballistically backmapped interplanetary data for CR 2053 together with a synoptic map of open field lines estimated using a PFSS model. As we are comparing with solar synoptic maps labelled with Carrington longitude, the backmapped solar wind parameters are shown with time increasing from right to left. Panel (a) shows solar wind speed. The dates at which higher-speed features are indicated in Figure 2(d) are attached to the corresponding backmapped features to help orient the reader. Panel (b) shows the sense of the radial interplanetary magnetic field (black) and the dominant strahl pitch angle (blue). Orange arrows compare the sector boundaries seen at $A C E$ with the intersection of the modeled HCS and the ACE footprint trace. Panel (c) shows the proton temperature in the solar wind, together with the "expected temperature." Panel (d) shows oxygen and carbon "freeze-in" temperatures. The interplanetary data sets are from $A C E$, at a cadence of $1 \mathrm{hr}$. Panel (e) shows a PFSS synoptic plot, with the boundaries of coronal holes and active regions, and the location of the modeled heliospheric current sheet from Figure 1 superimposed. The PFSS model's dashed white lines represent positive (away from the Sun) open magnetic flux and the solid white lines represent negative (toward the Sun) open magnetic flux. The magnetic flux is plotted from the photosphere up to a source-surface boundary at 2.5 solar radii. As a guide to the eye, yellow boxes indicate the backmapped footprint of the short-lived fast flow intervals and red boxes indicate the footprints of the fastest long-duration solar wind flows.

source-surface boundary at 2.5 solar radii. At lower latitudes the plotted lines originate in regions of high flux density including relatively localized boundaries of coronal holes and active regions, while at higher latitudes where there is no strong variation in flux density the footprints of the field lines are distributed throughout the polar coronal holes. The flow data for the solar wind are presented using the same longitude (time) convention as the synoptic plots.

The simple ballistic backmapping technique maps some flows that are observed at different times at L1 to the same Carrington longitude (e.g., the feature labelled 02/26 in Figure 3(a)). Similar artefacts of the ballistic backmapping process can be seen for example in Plate 2 of Neugebauer et al.
(1998). We suggest that the results are accurate enough to be useful, but not strictly accurate. This may be a consequence of applying the assumption of a constant wind speed during transit to L1, to plasma that in reality will change speed during transit at the boundaries of two flows of different speeds (as occurs on a large scale in stream interaction regions).

Broadly speaking, the fastest plasma flows in the solar wind map back to the longitudes of the three coronal holes mentioned earlier (indicated in Figure 3 by red boxes), while the slowest solar wind maps back to regions of quiet Sun (with neither active regions nor coronal holes). In the case of $\mathrm{CH} 1$, open field lines are shown in Figure 3 reaching up to the red box that represents the footprint of fast wind. PFSS field lines in our synoptic plot are not shown originating in the other equatorial coronal holes, but more detailed PFSS modeling (not shown) supports the view that these (rather than the polar holes) are the sources of the fast flows at $A C E$. The tendency for the backmapped peak flows to appear to the east of the corresponding coronal hole in the cases of $\mathrm{CH} 2$ and $\mathrm{CH} 3$ is due to longitudinal expansion of the coronal hole flux, according to the PFSS models. This result is consistent with the interpretation of Luhmann et al. (2009) and He et al. (2010b).

One measure of how well the backmapping technique worked is to compare the longitudes of the backmapped sector boundary crossings with the projected crossings of the HCS footprint and the $A C E$ footprint. The backmapped sector boundaries are at $318^{\circ}, 206^{\circ}, 162^{\circ}$, and $68^{\circ}$, while the WSO HCS footprints cross the $A C E$ footprint at $337^{\circ}, 225^{\circ}, 151^{\circ}$, and $68^{\circ}$. The orange arrows of Figure 3 illustrate this comparison, including a dashed arrow to highlight a possible brief sector boundary crossing at $148^{\circ}$. The estimated error in source longitude for the ballistic backmapping technique is often cited as $\leqslant 10^{\circ}$ (Nolte \& Roelof 1973). In our case the disagreements are $19^{\circ}, 19^{\circ}, 11^{\circ}$, and $\sim 0^{\circ}$, perhaps indicating additional uncertainties contributed by the PFSS modeling.

The sector boundary at $68^{\circ}$ clearly separates outflow plasma from a coronal hole (high speed, high proton temperature, and low freeze-in temperatures) from plasma of a different source (slower, lower proton temperature, and higher freeze-in temperature).

In addition to the coronal hole flows, there are a number of shorter-lived intermediate-speed flows at backmapped Carrington longitudes that roughly coincide with active regions, for example at Carrington longitudes of roughly $40^{\circ}, 135^{\circ}, 220^{\circ}$, and $290^{\circ}$. These are indicated in Figure 3 by yellow boxes. The PFSS model shows open flux reaching up from the boundaries between $\mathrm{CH} 1 / \mathrm{AR} 1, \mathrm{CH} 2 / \mathrm{AR} 4$, and $\mathrm{CH} 3 / \mathrm{AR} 5$ to yellow boxes representing the footprints of intermediate-speed flows. However, the weak signature of intermediate-speed flow that maps to $\sim 220^{\circ}$ does not appear to be linked by open flux to a coronal hole/active region boundary. We note that He et al. (2010b) examined the signature of intermediate-speed flow on February 26 and share our conclusion that it could be traced to the edge of AR4 using a backmapping/PFSS approach.

In the following, we will discuss the sources of the fast wind from coronal holes and then the source of the shorter intervals of intermediate-speed wind.

\section{FAST WIND FROM CORONAL HOLES}

As noted in the introduction, there have been studies of outflow from polar coronal holes, but there has been little coronal spectroscopic work on equatorial coronal holes, 
concentrating in particular on the coronal hole itself as opposed to the boundary or the jets and bright points. In particular, the range of size scales of structures has not been studied.

A popular model for outflow of solar wind from coronal holes was initially proposed by Fisk (2003), and involves open magnetic flux in the coronal hole undergoing repeated footpoint interchange reconnection with small loops in the coronal hole.

In order to examine this hypothesis, we present observations made by the EIS on board the Hinode spacecraft (Kosugi et al. 2007). The EIS instrument, described by Culhane et al. (2007), is a scanning slit spectrometer observing in two wavebands in the EUV: $170-210 \AA$ and $250-290 \AA$. The spectral resolution is equivalent to approximately $34 \mathrm{~km} \mathrm{~s}^{-1}$ per pixel for the $195 \AA$ emission line, which allows velocity measurements of a few $\mathrm{km} \mathrm{s}^{-1}$. The temperature coverage ranges from 0.05 to $20 \mathrm{MK}(\log T=4.7-7.3)$ with a spatial resolution of close to 1-2 arcsec, depending on the instrument mode.

The XRT (Golub et al. 2007) on Hinode was used to analyze the dynamics of the bright points in the coronal hole. We analyzed data from the Al_mesh filter, with a time cadence of approximately $80 \mathrm{~s}$.

The approximate coverage of a Hinode observation of coronal hole $\mathrm{CH} 1$ on 2007 February 12 is indicated on a fullSun STEREO-EUVI plot in Figure 4(a). The larger dotted red box indicates the coverage of the XRT image and the smaller solid red box indicates the coverage of the EIS raster. XRT produces images at $\sim 80 \mathrm{~s}$ cadence, while EIS took about $4.5 \mathrm{hr}$ to generate the raster. Figure 4(b) shows a rectangular EIS map of Doppler shift velocity, showing where plasma emitting in the Fe XII line (195 $\AA$, the same wavelength that is used in the STEREO image) is travelling toward or away from the Sun. Figure 4(c) shows a zoom covering a region about 100 arcsec square, which shows individual pixels corresponding to coverage of areas 2 arcsec square, corresponding to roughly $1500 \mathrm{~km}$ square. The zoom region is chosen to be well away from the coronal hole/active region boundary. Figure $4(\mathrm{~d})$ is a Hinode-XRT image, which confirms that any X-ray-emitting plasma is confined to very small scales and low fluxes in the zoom region. The EIS coronal hole data contain many smallscale features, ranging in size from $\sim 20 \operatorname{arcsec}$ down to the limit of resolution. Some show little flow, some have redshifted spectra, and more have blueshifted spectra indicating upflows. The peak measured upflow speed is $\sim 32 \mathrm{~km} \mathrm{~s}^{-1}$. EIS intensity data (not shown) indicate that the redshifted spectra tend to come from brighter sources, which we interpret as small closed coronal loops that are filled with hot plasma that is cooling, and thus the loops exhibit redshifted drainage downflows.

The smallest features may correspond to newly emerging loops with footpoint spacing of the order of $1500 \mathrm{~km}$. Larger features occupy tens of pixels and may represents loops that have footpoint spacing of 10-20 arcsec, i.e., $15,000-30,000 \mathrm{~km}$. If their heights are roughly half their footpoint spacing, then these lie near the bottom end of the height range for loops that Feldman et al. (1999), Fisk (2003), and Gloeckler et al. (2003) proposed to be involved in the release of solar wind from coronal holes. Thus they are expected to have freeze-in temperatures toward the lower end of the range $0.8-1.5 \mathrm{MK}$. The simple linear relationship between loop height and temperature assumed by Gloeckler et al. (2003) has temperatures of 1.0-1.1 MK for
$15,000-30,000 \mathrm{~km}$ loops. It predicts a temperature of $0.91 \mathrm{MK}$ for loop heights of $1500 \mathrm{~km}$, only slightly cooler than for $15,000 \mathrm{~km}$ loops. This length scale is at the lower edge of applicability, and future work may be needed to improve accuracy for small loop heights.

Figure 5 shows the characteristics of the solar wind measured at $\mathrm{L} 1$ for coronal holes $\mathrm{CH} 1, \mathrm{CH} 2$, and $\mathrm{CH} 3$, using the same format as Figure 2. It is clear that in each case there is an interval of roughly two days where the freeze-in temperature is consistently below $1 \mathrm{MK}$. During these intervals, the solar wind speed is above $600 \mathrm{~km} \mathrm{~s}^{-1}$, except for $\mathrm{CH} 3$ where the speed is above $500 \mathrm{~km} \mathrm{~s}^{-1}$. The average/lowest freeze-in temperatures are $0.87 / 0.77 \mathrm{MK}$ for $\mathrm{CH} 1,0.92 / 0.85 \mathrm{MK}$ for $\mathrm{CH} 2$, and $0.96 / 0.88 \mathrm{MK}$ for $\mathrm{CH} 3$ (where the average is calculated for the interval below $1 \mathrm{MK}$ ). As noted above, such values are expected for loop heights similar to those that we propose have been seen by Hinode-EIS.

Further evidence in support of the identification of these solar wind flows with an origin in the respective coronal holes is provided by the IMF (Figure 5(b)), which is directed outward from the Sun in panels (a) and (b) but inward in panel (c), as expected for $\mathrm{CH} 1, \mathrm{CH} 2$, and $\mathrm{CH} 3$ (see Figure 1), and the dominant component of the electron strahl in the solar wind, which is consistently directed outward from the Sun during these times.

Figure 5 shows that although the speed and freeze-in temperatures are consistent during the highlighted intervals, the early part of each interval shows stronger magnetic field, higher proton density, and longer periods with proton temperature in excess of "expected" values. The alpha/proton density ratios typically lie between $2 \%$ and $4 \%$, which is somewhat lower than the "typical" value for outflows from coronal holes established by Borrini et al. (1983), and they do not conform very closely to the statistical trend of higher ratios for higher speeds, showing lower ratios than expected for fast flows (Kasper et al. 2007). The pale green shaded area corresponds to the estimated time during which the EIS raster of Figure 4(b) was collected, taking account of ballistic backmapping. The zoom in Figure 4(c) corresponds to the later part of the green banded area, where the freeze-in temperature unequivocally shows values below $1 \mathrm{MK}$ that have previously been described in the literature as indicative of a coronal hole source.

The Fe/O ratio is an indicator of FIP bias, i.e., the systematic enrichment of elements with low FIP relative to elements with high FIP that is seen in the corona and solar wind, compared to their photospheric abundances. The relative enrichment is typically higher $(\sim 4)$ in slow solar wind than in fast solar wind $(\lesssim 2)$ and shows a systematic monotonic variation (Aellig et al. 1999). The variability of $\mathrm{Fe} / \mathrm{O}$ abundance ratio is typically much larger in the slow wind than in the fast wind. The $2 \mathrm{hr} \mathrm{Fe} / \mathrm{O}$ ratios measured by $A C E$ for $\mathrm{CH} 1, \mathrm{CH} 2$, and $\mathrm{CH} 3$ are $0.05-0.08$ (not shown), consistent with FIP bias $<2$.

The conditions are not especially stable during these intervals, including in parameters whose values are thought to be indicative of the coronal source and unlikely to be modified in transit, such as the alpha/proton density ratio and the freeze-in temperatures.

\section{INTERMEDIATE-SPEED WIND FROM CORONAL HOLE/ACTIVE REGION BOUNDARIES}

One suggested type of source for "slow" solar wind is the boundary region between coronal holes and active regions. 

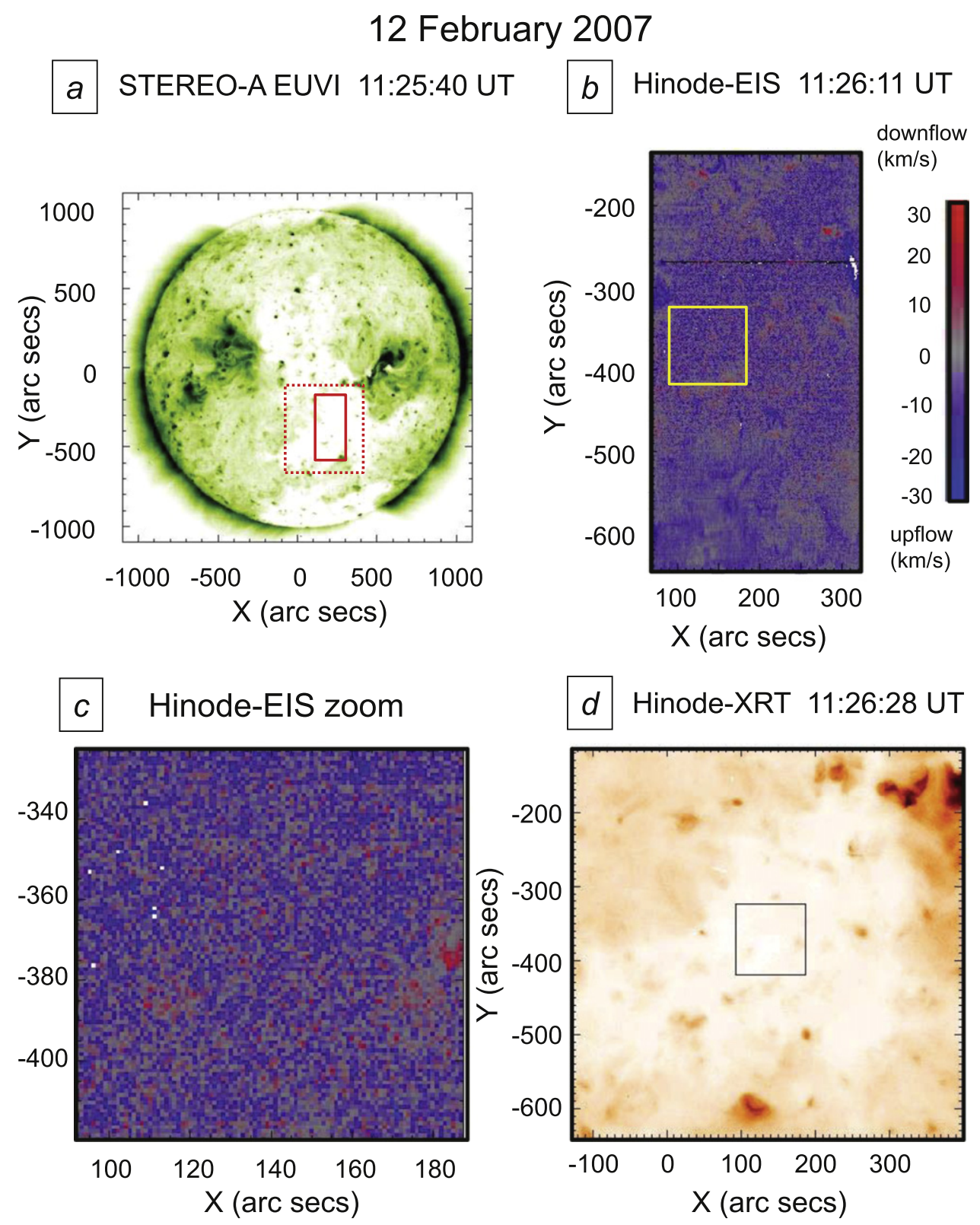

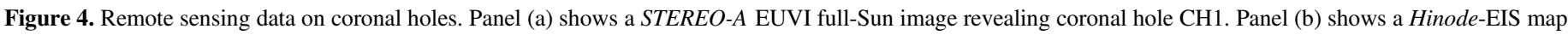

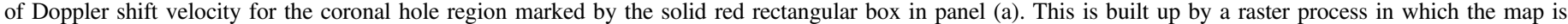

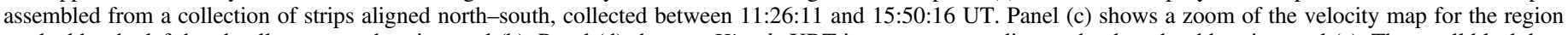

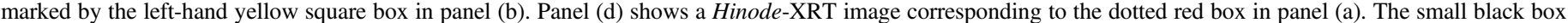
corresponds to the area covered in panel (c).

Here we examine three intervals, each lasting about 1 day, during which the solar wind speed at L1 is significantly elevated by about $50-70 \mathrm{~km} \mathrm{~s}^{-1}$ relative to the preceding and the following days. We present relevant solar data in Figure 6 and solar wind data measured at L1 in Figure 8. We present similar data in Figures 7 and 9 for cases of isolated active regions.

Figure 6 shows the STEREO EUV full-Sun images for context and Hinode-EIS maps of Doppler shift velocity for two examples of active regions with neighboring near-equatorial coronal holes to the west of the active regions, specifically $\mathrm{CH} 1 / \mathrm{AR} 1$ and $\mathrm{CH} 2 / \mathrm{AR} 4$. The velocity maps each cover regions approximately 250 arcsec square, larger than Figure 4(c). In each case there are well defined patches of upflowing and downflowing plasma, on a typical scale of a few tens of arcseconds, in contrast to the more intermingled, much smaller-scale regions in the example of a coronal hole discussed above. The strongest upflows are seen on the east side of each active region, which is the side adjacent to the coronal hole in each case. As shown in Figure 8, in situ data at L1 appear to show corresponding flow enhancements.

In Figure 6, left column, the 2007 February 11 EIS spectral scan focuses on the sigmoidal active region AR 10938 (AR1). An area of concentrated fast upflowing plasma is seen on the top left of the image, on the side of the active region neighboring the coronal hole. The blueshifted upflows originate mainly from the boundary of the redshifted closed loops, in areas where sudden changes in loop connectivity occur, 


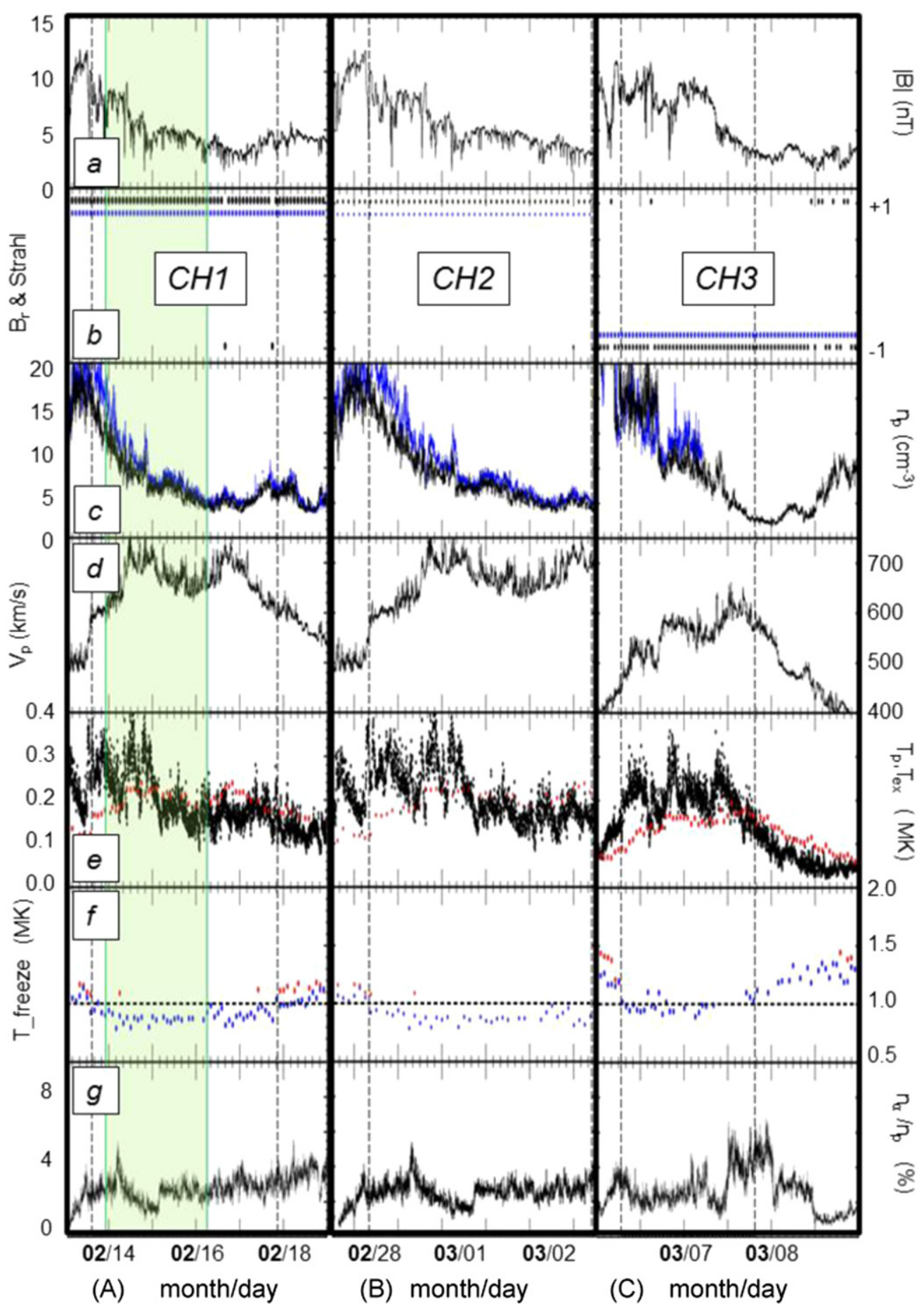

Figure 5. Interplanetary data associated with coronal holes $\mathrm{CH} 1, \mathrm{CH} 2$, and $\mathrm{CH} 3$, presented in three panels that each show a 3 day interval using the same format as Figure 2. In each panel, the pairs of vertical dotted lines delimit the period in which the "freeze-in temperature" of the solar wind is less than $1 \mathrm{MK}$, considered to be a signature of coronal hole origin. During these times the solar wind speed always exceeds $500 \mathrm{~km} \mathrm{~s}^{-1}$; indeed for CH1 and CH2 it is usually greater than $600 \mathrm{~km} \mathrm{~s}$. The region of the Sun observed by Hinode-EIS presented in Figure 4(b) was observed after the region crossed the solar central meridian. The green shading in panel (a) corresponds to the time interval that backmaps to the central meridian, when the region seen later by Hinode was at the central meridian.

separating the closed loops of the active region from larger scale externally connected loops or open field lines. The EIS observations used the Fe XII emission, corresponding to plasma at $1.2 \mathrm{MK}$ (not to be confused with electron freeze-in temperature discussed elsewhere). The peak bulk upflow velocity measured in this case was $23 \mathrm{~km} \mathrm{~s}^{-1}$. The region is characterized by relatively short loops connecting dominantly within the active region.
Figure 6 in the right column shows the well studied active region AR 10942 (AR4) on 2007 February 20, which has been discussed by several authors as noted in the introduction, including Sakao et al. (2007), Harra et al. (2008), Baker et al. (2009), and He et al. (2010b). Both AR1 and AR4 shown in the figure have been plotted on the same velocity scale, and hence it is very clear that AR4 shows much more distinct and stronger upflowing plasma. Sakao et al. measured the motion of bright 
AR1: 11 February 2007

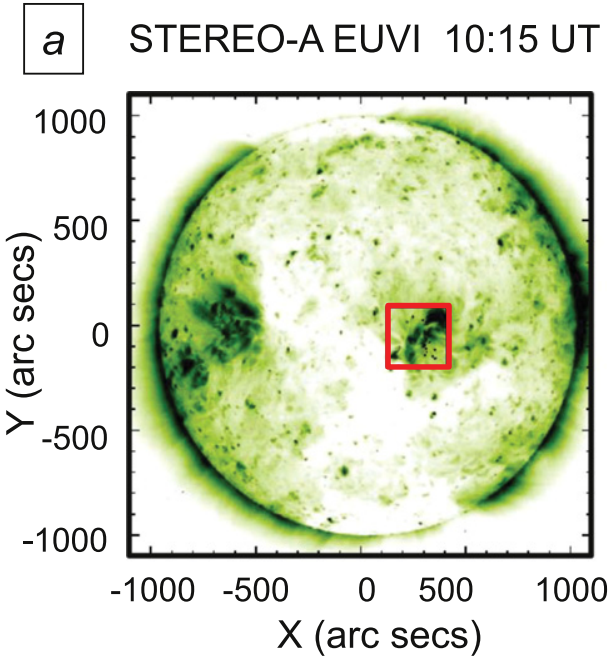

C Hinode-EIS 10:13:49 UT

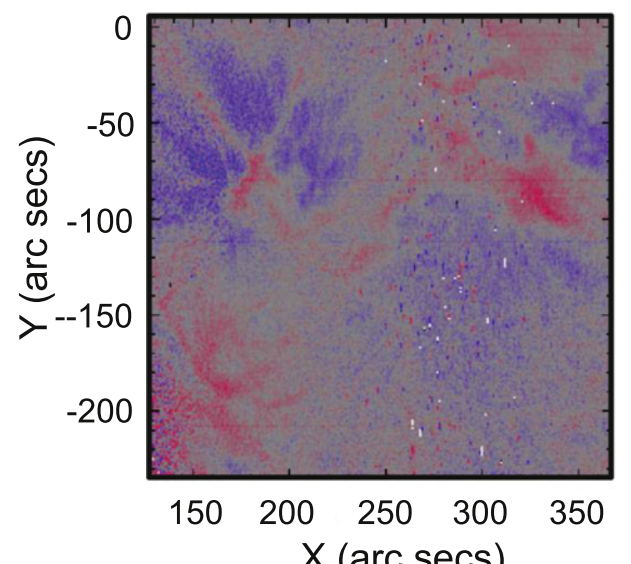

\section{AR4: 20 February 2007}

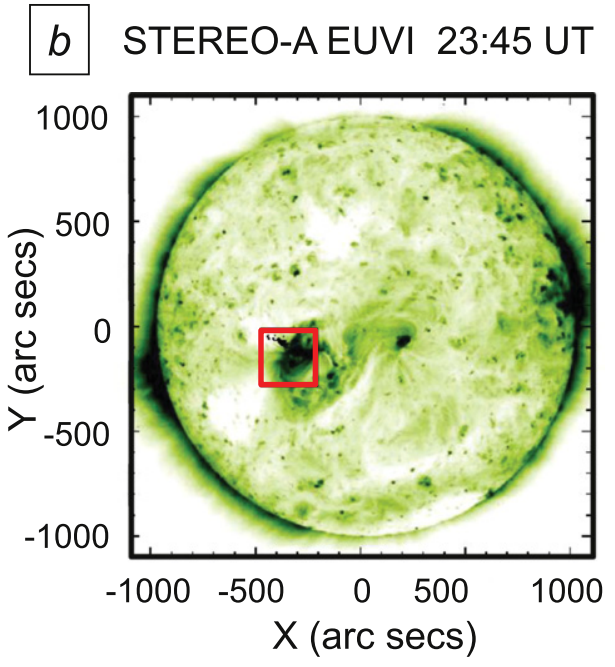

d Hinode-EIS 23:45:15 UT

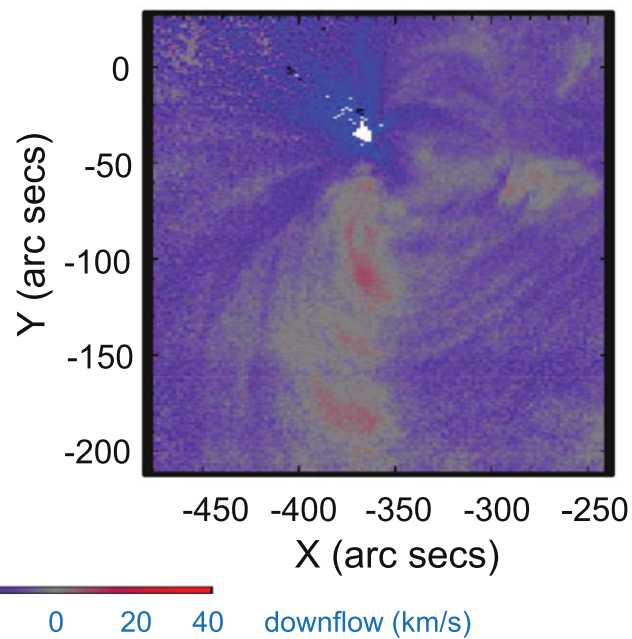

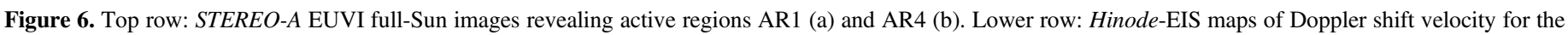
active regions marked by the corresponding rectangular boxes in the panels above (the February 20 data are reproduced from Harra et al. 2008).

features transverse to the line of sight observed in Hinode-XRT X-ray data. The bright features appeared to travel along a bundle of fan-like magnetic field lines originating from the east side of the active region (adjacent to the coronal hole) with typical transverse speeds of $140 \mathrm{~km} \mathrm{~s}^{-1}$. The flows persisted continuously for at least three days (February 20-22). Harra et al. (2008) explored the magnetic orientation and structure of the upflowing region reported by Sakao et al. and found it to be related to large-scale loops reconnecting with a smaller bipolar active region lying several degrees of longitude away (our AR3). He et al. (2010b) used data from Hinode-XRT and TRACE to show that in one emission structure the soft-X-rayemitting plasma outflow was intermittent, occurring roughly every 20 minutes, with flow speeds sometimes exceeding $200 \mathrm{~km} \mathrm{~s}^{-1}$. He et al. (2010a) used Hinode-SOT data to observe chromospheric jets appearing with similar cadence to the XRT outflows, which were demonstrated to be upflows using evidence of blueshifts in He II observations. However, magnetic reconnection along QSLs is also expected to be intermittent (Baker et al. 2009).

Figure 7 shows the STEREO EUVI data and Hinode-EIS Doppler velocity data for AR2 and AR3. In both these examples there are no significant enhancements seen in the in situ speeds shown in Figure 9. The format of Figure 7 is the same as Figure 6. AR2 has a coronal hole to the west of the region, but the active region and the coronal hole are on opposite sides of the heliospheric plasma sheet (see Figure 3). In addition, the separation of the active region and coronal hole is larger than those for the $\mathrm{CH} 1 / \mathrm{AR} 1, \mathrm{CH} 2 / \mathrm{AR} 4$, and $\mathrm{CH} 3 /$ AR5 cases discussed above. There are plasma upflows, with a peak velocity of $18 \mathrm{~km} \mathrm{~s}^{-1}$, but the structures of the outflows appear different, less fan-like than in, e.g., AR4. AR3 is the small active region that is connected to AR4. The peak upflow in this case is $17 \mathrm{~km} \mathrm{~s}^{-1}$.

AR5 and AR6 represented the most complex magnetic configuration in this Carrington Rotation, including two 
AR2: 15 February 2007
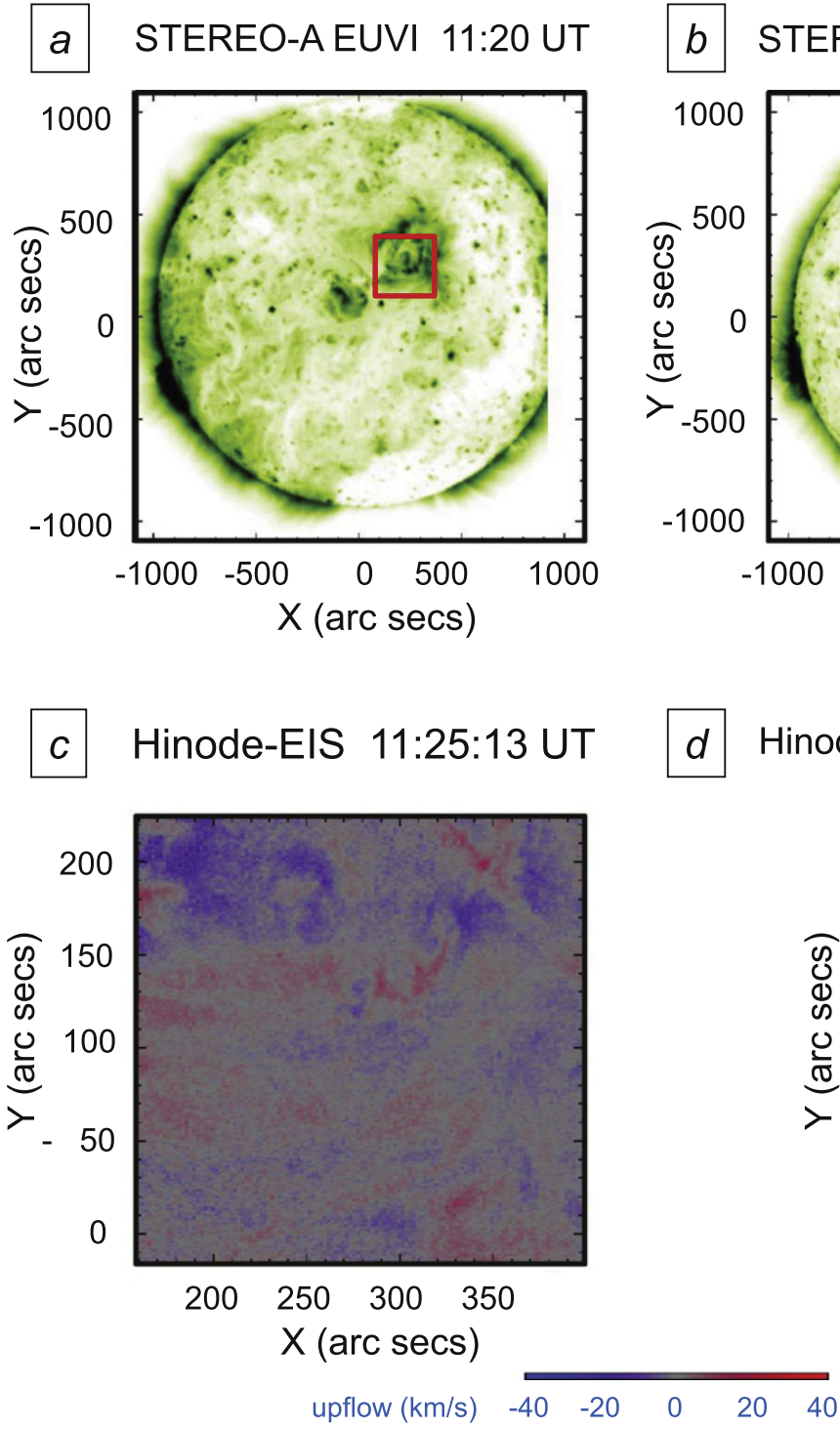

AR3: 22 February 2007

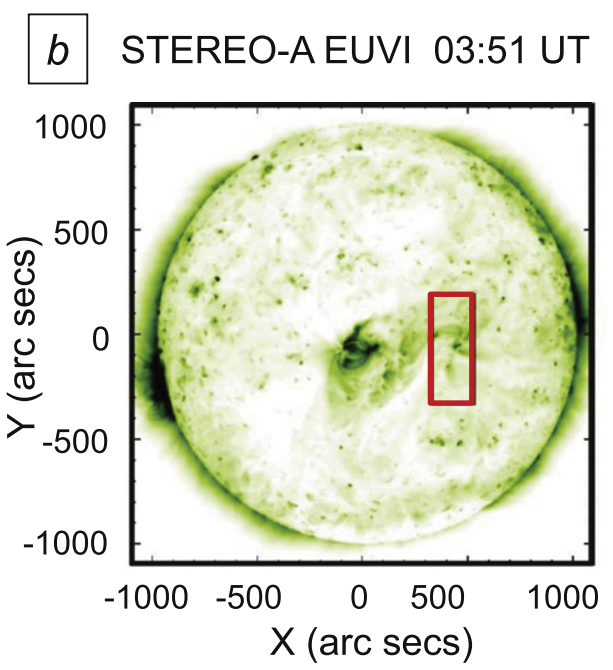

Hinode-EIS 03:53:30 UT

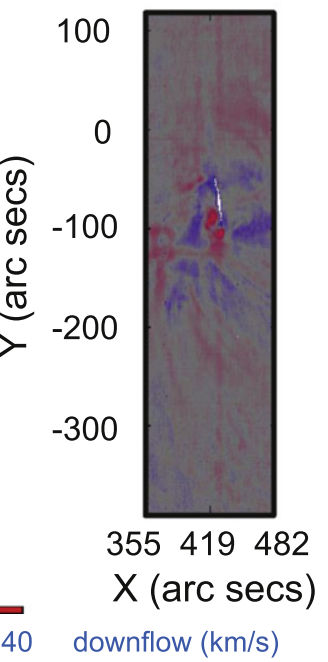

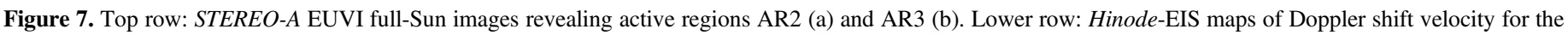
active regions marked by the corresponding rectangular boxes in the panels above.

anemone (tripolar) structures, which should include highaltitude coronal nulls. We cannot show spectral observations of the complex AR5 active region and coronal hole because EIS did not observe the interface of this complex with the coronal hole.

Figure 8 presents solar wind data from L1 in a similar format to Figures 2 and 5. The three panels marked (A), (B), and (C) correspond to intervals of 1 day that backmap to the vicinities of the coronal hole/active region pairs $\mathrm{CH} 1 / \mathrm{AR} 1, \mathrm{CH} 2 / \mathrm{AR} 4$, and $\mathrm{CH} 3 / \mathrm{AR} 5$ respectively. Pale green shading in panels (A) and (B) indicates the estimated times during which the corresponding EIS observations (Figure 6) were made, after applying ballistic backmapping. In each case the magnetic field is directed in the same sense as in the neighboring coronal hole. The dominant strahl motion is outward in each case (showing $0^{\circ}$ pitch angles for (a) and (b), and $180^{\circ}$ pitch angle for (c)), consistent with a very similar magnetic connection to the Sun as for the nearby coronal holes.
Pairs of vertical dotted lines delimit intervals of enhanced solar wind speed in relation to the preceding and following periods. The average speeds in the intervals are 514, 394, and $430 \mathrm{~km} \mathrm{~s}^{-1}$ respectively so that the first case might be regarded as "fast wind." The proton temperatures are consistently higher than the "expected values" for the observed flow speeds. The freeze-in temperatures correspond fairly well to expectation given the relationship between speed and freeze-in temperature, being at $1 \mathrm{MK}$ or below for case (a) and between 1 and $1.2 \mathrm{MK}$ for case (c). As was noted by He et al. (2010b), the charge state ratios of oxygen and carbon are not routinely available for case (b). This was due to low count rates (perhaps consistent with the particularly low proton density at this time); however, the $A C E$-SWICS team was able to support our study by generating reliable data on charge state ratio accumulated over $6 \mathrm{hr}$ rather than the usual $1 \mathrm{hr}$ (the points are plotted at the center of the accumulation interval) with the exception of carbon ratios between 06 and $18 \mathrm{hr}$ on February 26. 


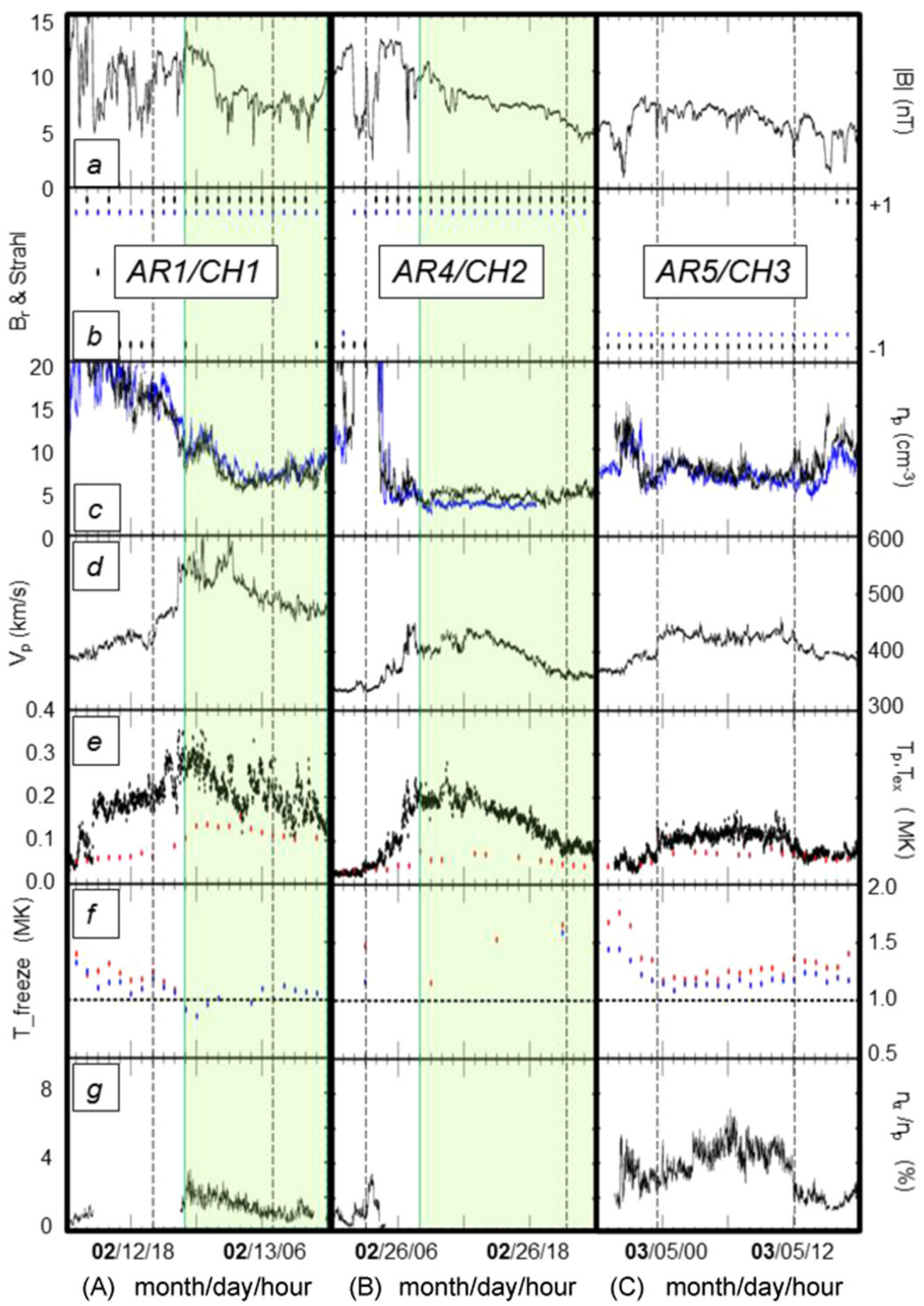

Figure 8. Interplanetary data associated with three active region/coronal hole boundaries, presented in the same format as Figure 5. Here the three panels cover the intermediate-speed solar wind streams at the interfaces of AR1/CH1, AR4/CH2, and AR5/CH3 respectively. The three panels each show intervals of 1 day, with the labels on the time axis describing month/day/hour. The pairs of vertical dotted lines delimit the period in which the solar wind speed is significantly enhanced relative to the previous and following days. The regions of the Sun observed by Hinode-EIS presented in Figure 6 were not observed while the regions crossed the solar central meridian. The green shaded regions in panels (a) and (b) correspond to the time intervals that backmap to the central meridian when the regions seen by Hinode were at the central meridian. In both cases the backmapped intervals end $\sim 8 \mathrm{hr}$ after the end of the plotted interval (not shown).

The ratio $n_{\alpha} / n_{\mathrm{p}}$ for AR1/CH1 is $\sim 2 \%$, which is lower than expected for a $>500 \mathrm{~km} \mathrm{~s}^{-1}$ flow (based on statistics in Kasper et al. 2007) similar to the situation with $\mathrm{CH} 1$. The slower flows in panels (b) and (c) would be expected to show a $n_{\alpha} / n_{\mathrm{p}}$ ratio of $\sim 2.5 \%$, but the ratio for the third event is around $4 \%$, similar to the highest values seen in the solar wind associated with its neighboring coronal hole. The ACE-
SWEPAM data do not show alpha/proton density ratios during the interval in panel (b), but the ACE-SWICS $n_{\alpha}$ data allow us to comment that the $n_{\alpha} / n_{\mathrm{p}}$ ratio is less than $2 \%$ during the unplotted interval in panel (b), similar to the earlier event in panel (a). In both cases the values are somewhat lower than those for the solar wind associated with their neighboring coronal holes. 


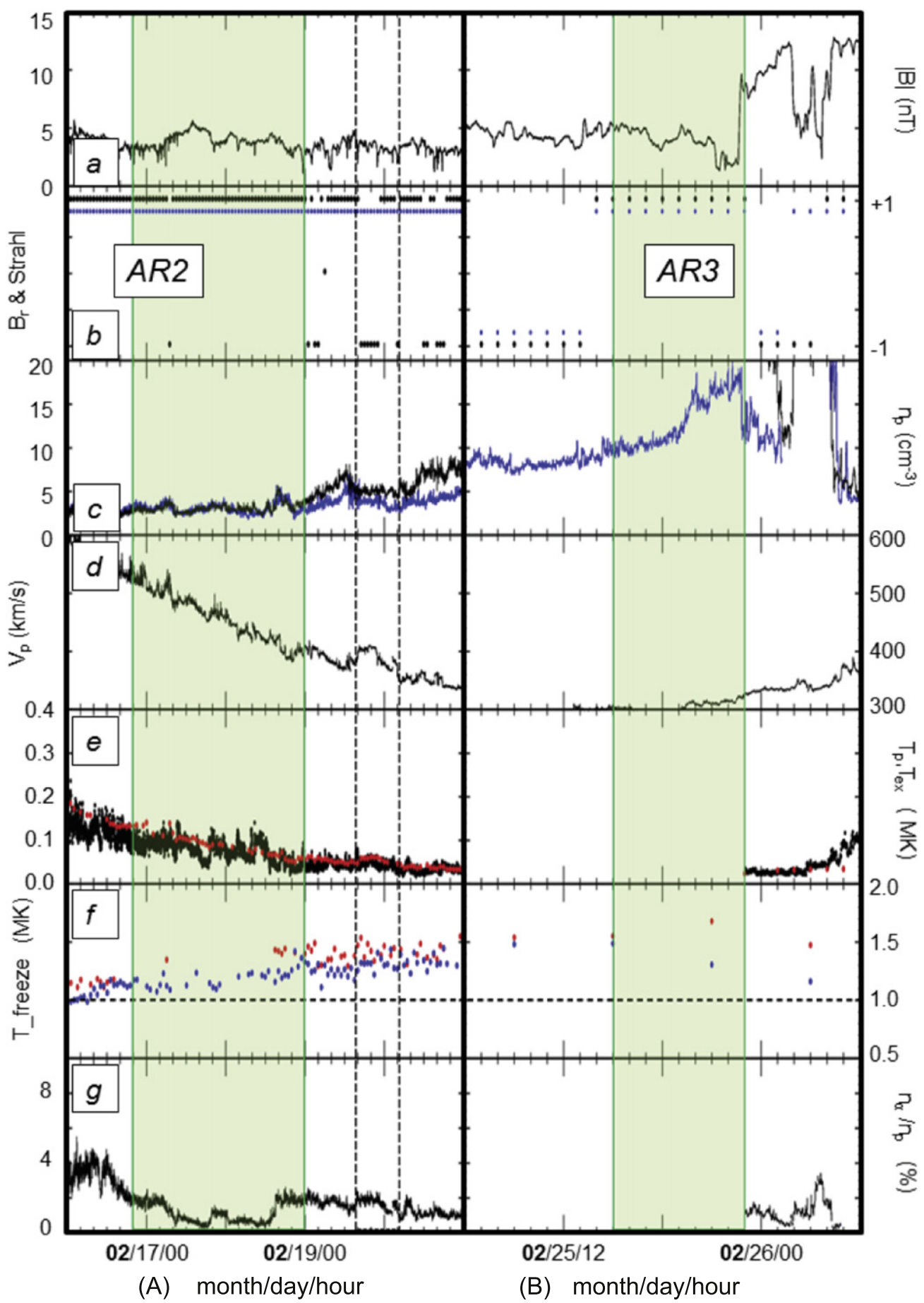

Figure 9. Interplanetary data associated with two further active regions (without neighboring coronal holes), presented in the same format as Figure 8. Here the two panels cover intervals associated with active regions AR2 and AR3, respectively. We suggest that the short flow enhancement indicated in panel (A) is probably due to interchange reconnection in the solar wind rather than a signature of fast outflow from an active region source.

Another in situ parameter of interest is the $\mathrm{Fe} / \mathrm{O}$ ratio. This parameter (not plotted) was not available for AR4/CH2. The $A C E 2 \mathrm{hr} \mathrm{Fe} / \mathrm{O}$ value was 0.08 for AR1/CH1 while it was 0.12 for AR5/CH3. The corresponding FIP bias values are $\sim 2$ and $\sim 3.3$, higher for the case of slower flow, following the general trend expected from Aellig et al. (1999), but not closely matching the FIP values expected for the observed flow speeds.

In Figure 9 we show the in situ data that backmap to the source-surface above active regions AR2 and AR3. There are no significant flow enhancements during the intervals (green shading) associated with Hinode observations in Figure 7. There is a short interval of speed enhancement in panel (A) (between dotted vertical lines), superimposed on a slow (sub $400 \mathrm{~km} \mathrm{~s}^{-1}$ ) solar wind flow, but no clear link via open flux to AR2 in Figure 3. Panel (B) shows no flow enhancements; in fact there are quite steady flows of $\sim 300 \mathrm{~km} \mathrm{~s}^{-1}$ during the interval associated with AR3. During the interval of flow enhancement in panel (A), the proton temperatures do not differ from the "expected" values, unlike the three cases in Figure 8, and the freeze-in temperatures are higher, at levels 
expected for an active region source, both before, during and after the flow enhancement. This conclusion is supported by the $\mathrm{Fe} / \mathrm{O}$ ratio (not plotted), which corresponds to FIP bias values larger than 3 . The ratio $n_{\alpha} / n_{\mathrm{p}}$ is $\sim 2 \%$, which is also consistent with an active region source, while the wind observed before and after typically has a slightly lower ratio. The IMF radial component before and after is positive, suggesting a connection to the southern hemisphere although AR2 is in the northern hemisphere. It switches direction at the boundaries of this flow feature, and for a handful of shorter-lived flow enhancements seen shortly before and after; however, the strahl direction remains steadily parallel to the local field throughout.

\section{DISCUSSION}

In our analysis we have studied a full Carrington Rotation to determine the range of solar wind sources that exist. We see periods of fast solar wind that are related to equatorial coronal holes, and periods where the slow solar wind has short velocity enhancements, about one day long at $1 \mathrm{AU}$, which are related to active regions. We will discuss the cases of both fast and slow wind.

\subsection{Fast Solar Wind}

There are three periods of fast solar wind during the Carrington Rotation, all related to coronal holes. The EIS data show a mix of red- and blueshifted plasma within a coronal hole, highlighting the coexistence of open and closed field within a coronal hole. The redshifted plasma is likely to be on closed loops, which are seen at different size scales, draining plasma as they are cooling. The blueshifted plasma is related to plasma that is free to flow upwards through open or large-scale loops. Our observations are broadly consistent with the picture of Fisk (2003), in which coronal holes exhibit a mixture of open field lines together with relatively small-scale $(30 \mathrm{Mm})$ closed loops, and in which the open field lines regularly reconnect with closed loops, causing downflows on newly formed loops and escaping upflows on newly open magnetic flux. Figure 10 illustrates this, highlighting where the upflow and downflow are seen in the EIS data.

The Fisk picture describes outflows only for magnetic flux that is reconnecting with loops on scales of tens of Mm, which might be expected to produce localized upflows in the EIS velocity maps near large loops rooted along supergranular boundaries. In fact this is what is seen in SUMER observations of polar coronal holes. SUMER observes cooler plasmas than EIS, with outflow associated with the chromospheric network structure (Hassler et al. 1999), and the pale blue band in Figure 10 attempts to illustrate this. However, the EIS velocity maps for a low-latitude coronal hole show wide coverage of blueshifted material with $80 \%$ of the area being covered in blueshifted pixels-this includes at size scales of $\sim 1 \mathrm{Mm}$. We interpret these widespread small-scale blueshifts as upflows on recently open flux tubes, as illustrated in the cartoon in Figure 10. Plasma at the temperature that EIS is observing is mainly in the corona so EIS will be observing at the altitudes where the open loops have expanded, explaining why we observe a large fraction of the raster to be blueshifted. EIS sees redshifted plasma that is interpreted as being hot, descending plasma at lower altitudes than the blueshifted plasma. The distribution of this redshifted plasma might be expected to outline the supergranular boundaries, but such a pattern is not
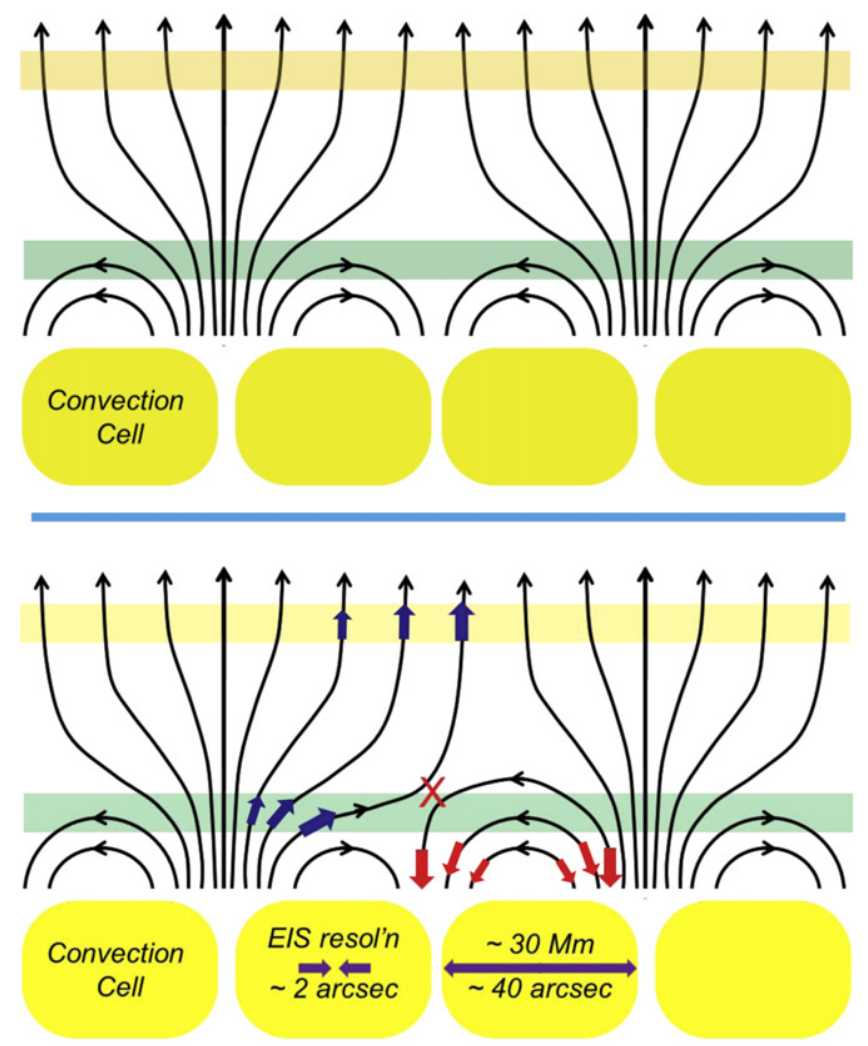

Figure 10. Illustrations in the upper and lower panels schematically show photospheric convection cells in yellow, with the magnetic fields of coronal holes above, including loops on supergranular scales of $30 \mathrm{Mm}$ and open magnetic flux reaching to higher altitudes. The horizontal brown band roughly indicates the altitudes of plasmas emitting at the wavelength used by HinodeEIS, while the lower green band illustrates the lower altitude at which chromospheric coronal hole plasmas have been observed by SOHO-SUMER in Ne XIII. SUMER observations (e.g., Hassler et al. 1999) typically showed upflows confined to the chromospheric network boundaries, i.e., on open flux near convection cell boundaries. The lower panel illustrates our suggestion of how the Fisk model of interchange reconnection leads to EIS observations of widespread blueshifts in the coronal hole (seen in Figure 4), in contrast with the more confined regions of upflow in the SUMER observations. Outflows at the higher altitudes seen by EIS, as shown by the blue arrows in the brown shaded region, are at altitudes where the open flux has expanded, in contrast to the open magnetic flux confinement at chromospheric altitudes. Downflows are predominantly expected on newly closed loops of a range of heights from granular to supergranular scales (see scales indicated in the lower panel), which appear redshifted as the plasma is draining. The corona is optically thin and hence we observe a superposition of the open and closed magnetic field regions.

readily apparent. Further studies are planned, and will use observations from modern high resolution instruments, such as those on board IRIS and SDO, alongside Hinode.

In addition, there is a remarkable variability in the coronal hole data in most parameters (density, velocity, proton temperature, and alpha/proton ratio), and to a smaller degree in the freeze-in temperature. The variability appears to be higher in coronal holes than in the periods of velocity enhancements of the intermediate solar wind. This seems to be opposite to the general perception of fast wind being significantly steadier than slow wind, but it may be characteristic of low-latitude coronal holes that exist in the activity belt. 


\subsection{Intermediate-speed Solar Wind}

We have highlighted three periods of intermediate-speed wind lasting about 1 day each, where the speeds are enhanced above the background speed, making them between fast and slow wind. These intermediate-speed wind streams represent wind speed enhancements of $40-100 \mathrm{~km} \mathrm{~s}^{-1}$ relative to the preceding and following days. The characteristics of the magnetic field and the strahl during these intervals are similar to those in the adjacent fast wind, but the oxygen freeze-in temperatures are systematically a little higher than in the neighboring fast wind streams, while also being lower than in the slower wind that precedes and follows these intervals. However, the conditions in each of these intervals also show quite strong individuality, with especially fast flows in the first, especially low density in the second, and rather slow flows and the highest alpha/proton ratios in the third. The EIS data for the active regions involved all show upflows and downflows, with the upflows tending to lie close to a coronal hole to the east of the active region as illustrated in Figure 3.

Each of these intermediate-velocity wind streams is associated with a different coronal magnetic field topology, as summarized in Figure 11. The first scenario (Figure 11(a)) is for the bipolar (sigmoidal) active region AR1 and its nearby coronal hole $\mathrm{CH} 1$. The coronal hole has the same polarity as the neighboring active region, allowing them to interact through small-angle "component reconnection." The block arrows highlight where the upflows (blue) and downflows (red) would be expected to be. The second scenario in Figure 11(b) is that of a tripolar active region (AR5) with a coronal hole (CH3). Again the coronal hole and active region have the same polarity, lying beside each other. In this scenario "standard" or "antiparallel" reconnection can occur between negative- and positive-polarity field, in addition to the small-angle reconnection already illustrated in panel (a). The quadrupolar case (Figure 11(c)) is the most complex and involves the two active regions (AR4 and AR3) and the coronal hole $(\mathrm{CH} 2)$. This is the situation that produces the fastest upflow on the disk as measured by EIS, but the fastest outflow measured in the solar wind itself was from the simpler scenario of the bipolar situation.

Component reconnection may occur between a magnetic flux loop in an active region containing a relatively highdensity plasma and adjacent flux containing a relatively lowdensity plasma that may be part of a much larger loop or an open field line in a coronal hole. The interchange reconnection effectively swaps the adjacent footpoints of the loops and will result in a flow of denser plasma driven by a pressure gradient from the former active region loop that is directed sunwards (downflow) to the new footpoint of the active region loop and anti-sunwards (upflow) along the larger loop. If the larger loop is sufficiently extended, or "open," the upflowing plasma can escape to form part of the solar wind. Numerical experiments by Bradshaw et al. (2011) suggest that the upflows driven by a pressure gradient could have supersonic speeds and predict spectral signatures in the upflowing plasma that are consistent with Hinode-EIS observations. The interchange reconnection in this picture is proposed to happen at high-altitude null points in the corona $(\sim 100 \mathrm{Mm})$, in contrast to the Fisk model for coronal outflows.

In order to explain the differing character of our three intermediate-speed solar wind events, we propose that reconnection along QSL or null points takes place at different altitudes in each case and connects magnetic flux in a coronal hole to active region loops of different scales, resulting in the differing freeze-in temperatures and correspondingly different solar wind speeds. For example, we would expect to be able to observe smaller-scale loops in AR1 than in AR4 or AR5 if the "loop height" model of the freeze-in temperature of solar wind is correct.

Although we have not performed detailed modeling, we suggest that the magnetic configuration in each of the three coronal hole/active region interfaces that we have presented is similar to that modeled by Baker et al. (2009), Del Zanna et al. (2011), Van Driel-Gesztelyi et al. (2012), Culhane et al. (2014), and Mandrini et al. (2014). In the last work a velocity enhancement similar to the three events described here was identified in the in situ data, and magnetic modeling linked this directly to the upflow regions from one of the active regions. We suggest that the intervals of enhanced solar wind velocity of $\sim 1$ day duration represent localized streams originating at active region/coronal hole interfaces, similar to the proposal of $\mathrm{He}$ et al. (2010b). However, we differ from $\mathrm{He}$ et al. by proposing that the outflows are occurring because of interchange reconnection at coronal rather than chromospheric altitudes.

We considered whether the fourth interval of intermediatespeed flow enhancement on February 19 might also be interpreted in terms of a coronal hole/active region interaction. AR2 might in principle interact on its western side with the nearby extension of $\mathrm{CH} 1$, which has predominantly positive magnetic field, or on its eastern side with the small coronal hole that has predominantly negative magnetic field. The location of the backmapped flow fits better with the latter case. The IMF observed during the enhanced flow interval points sunward, at first sight consistent with a source region of negative field. However, the direction of the strahl is parallel to the magnetic field, contrary to expectations for a source of negative field. Furthermore, as the strahl direction remains parallel before, during, and after the interval, it is more likely that the IMF has undergone interchange reconnection in the solar wind, resulting in the magnetic flux bending back on itself, in a similar way to that illustrated in Crooker et al. (2004). The flow perturbations might be dynamical effects associated with the magnetic tension on recently reconnected magnetic field lines, though we consider it outside the scope of this paper to investigate this hypothesis in detail. The lack of significant variation in composition parameters (freeze-in temperature, FIP bias, $n_{\alpha} / n_{\mathrm{p}}$ ratio) would also be consistent with an interpretation in which this is an interval of typical slow wind, with no special association with AR2, that experiences interchange reconnection in the solar wind, far from the Sun. Similarly we did not find any specific evidence suggesting that AR3 was a direct source for solar wind observed at L1.

\section{CONCLUSIONS}

We have used a combination of solar remote sensing data and in situ solar wind data from L1 to investigate sources of the solar wind reaching L1 during Carrington Rotation 2053. Figure 12 provides a summary of the phenomena in Carrington Rotation 2053 that we have discussed, and illustrates our assessment of the different sources of the solar wind during Carrington Rotation 2053 using a summary cartoon. This interval during solar minimum was chosen for its relatively few isolated active regions and lack of ICMEs, which allow relatively uncomplicated associations to be made between solar 

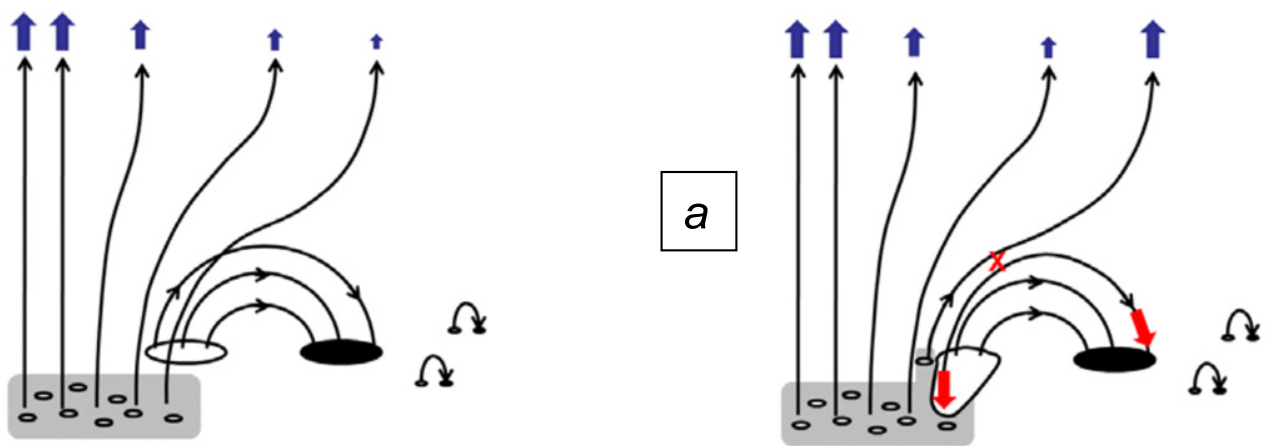

CH1/AR1

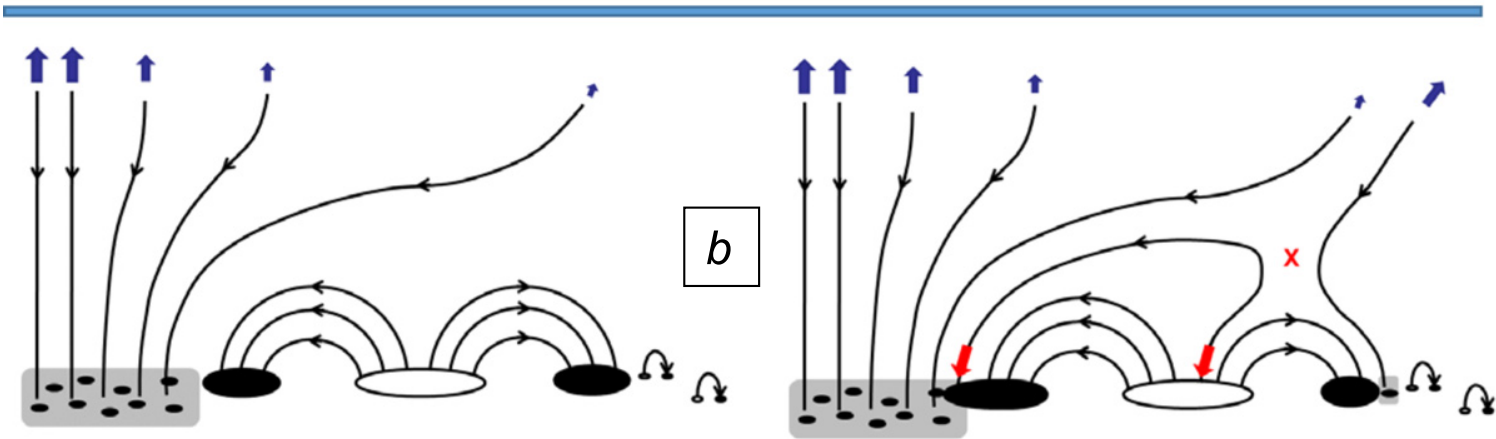

CH3/AR5
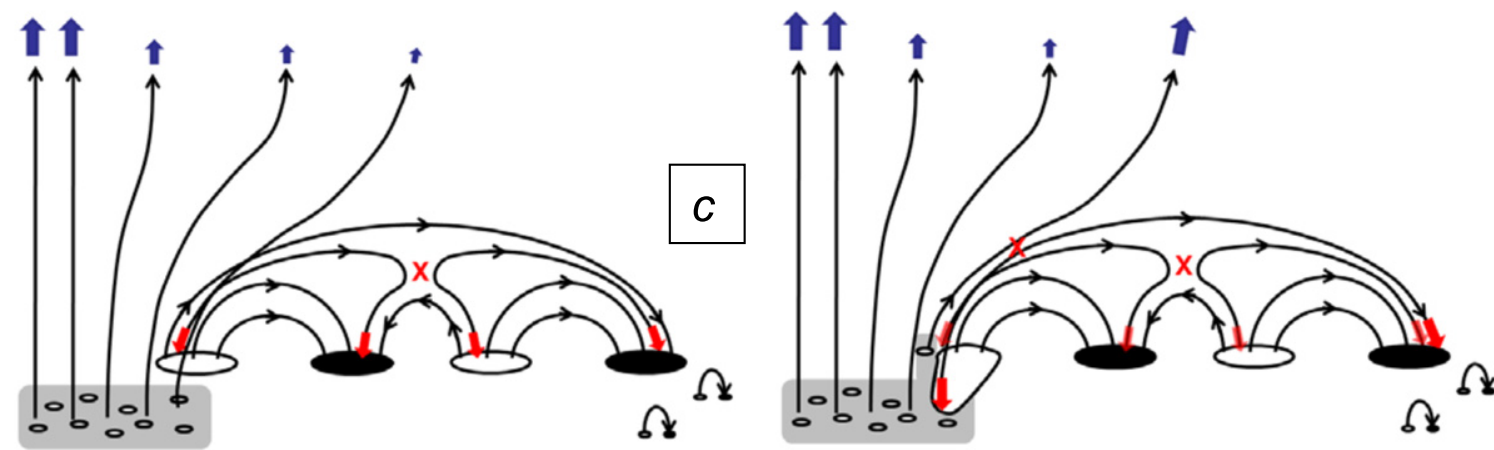

\section{CH2/AR3/AR4}

Figure 11. Illustration of interchange reconnection scenarios between a coronal hole and a neighboring active region complex, for which the magnetic polarity of the coronal hole is the same as that of the adjacent part of the active region. Panels (a), (b), and (c) illustrate bipolar, tripolar, and quadrupolar active region configurations respectively. The left-/right-hand figure in each panel shows the magnetic topology before/after interchange reconnection. Blue arrows indicate upflowing plasma on open field lines. The red arrows indicate downflowing plasma on closed loops. The gray patches indicate areas of coronal holes. In all cases, component reconnection occurs where a favorable situation arises. The possibility of magnetic flux from coronal holes reconnecting with the lower central loop in panel (c) also exists, but it is not illustrated. The composition of the upflowing plasma is expected to depend on the age of the newly opened active region loop. In the case of the upper large loop in panel (c), this is more complicated because that loop may have been produced by reconnection within the active region complex.

wind features and potential solar sources. We had EIS spectroscopic data coverage for six active regions and a coronal hole during this Carrington Rotation. The upflowing plasma in the corona so frequently measured by Hinode-EIS has often been linked to solar wind sources, but care is required because not all upflows are in fact outflows. In this work we aim to explore this further by studying a range of magnetic configurations in which plasma outflow can occur.

In the coronal hole analysis, we found that although the plasma shows dominant upflows there was also a range of size scales of redshifted plasma, indicating closed loops of differing size scales. We suggest that the description of Fisk (2003) may need to be extended to include interchange reconnection between open flux and coronal hole loops on a range that scales from the granular to the supergranular.

We examined isolated intervals of solar wind that have characteristics between slow wind and fast wind, and durations of about 1 day at $1 \mathrm{AU}$. Using backmapping and PFSS analysis we find that these are linked to active regions that lie to the west of a neighboring coronal hole, and in situations where 


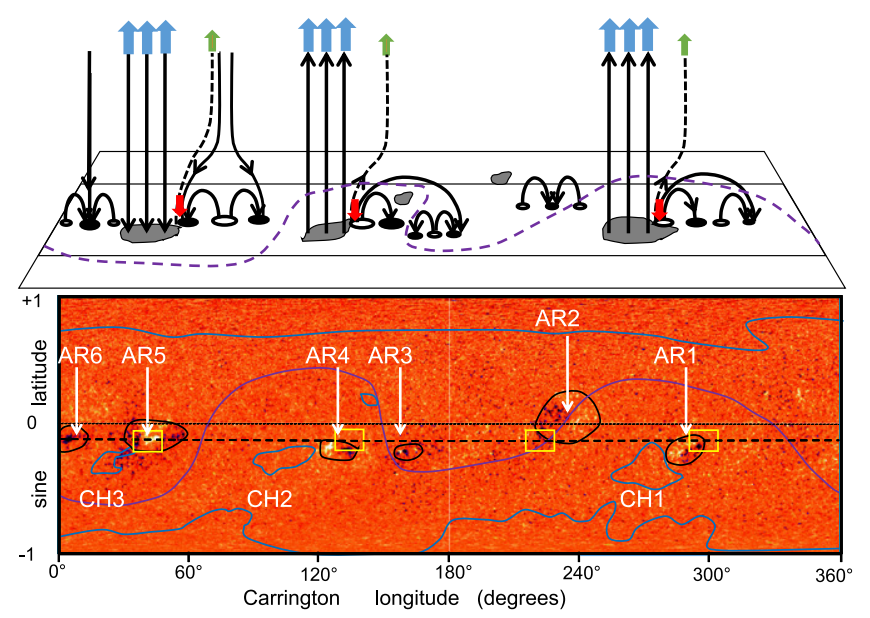

Figure 12. Illustration of our interpretation of the overall situation in CR 2053. The lower panel is a combination of the lower panels of Figures 1 and 3, illustrating the relative locations of the active regions and coronal holes that are discussed here. The upper panel is a cartoon illustrating the magnetic configurations of the active regions and coronal holes, and the key plasma flows, namely the coronal hole outflows (blue arrows) and the coronal hole/ active region boundaries' outflows (green arrows) and downflows (red arrows).

both active region and coronal hole lie on the same side of the HCS. This was the situation for bipolar, tripolar, and quadrupolar active regions during this Carrington Rotation. This indicates that the complexity of the active region is not critical to providing additional solar wind components. We illustrate the means by which interchange reconnection can occur in each of these magnetic configurations.

The common factor linking our interpretations of the sources of solar wind during the intervals analyzed is interchange reconnection. We propose an alternative interpretation to the model of He et al. (2010b) of chromospheric jets as the source of the solar wind in such an event. The reasons are that although we observe upflows in the active regions continuously, we do not always observe their signature in the solar wind. In addition the composition measurements made show coronal-type plasma, which cannot come directly from the chromosphere. The chromospheric flows are an important element to understanding how the atmosphere is formed and sustained, but it is not guaranteed that they will form part of the wind.

An improved understanding of the conditions necessary for active regions and adjacent near-equatorial coronal holes to feed plasma into the solar wind will be an important contribution to the mission operations planning for the upcoming Solar Orbiter mission. Solar Orbiter will be reliant on models to guide pre-planned observations to the solar wind source regions on the Sun that are most likely producing the solar wind measured at the spacecraft.

We gratefully acknowledge the ACE, STEREO, and SOHO instrument teams, the Wilcox Solar Observatory, and NASA's CDAWeb. In particular, we thank Sue Lepri and colleagues for careful examination of $A C E$-SWICS data around the 2007 February 26 period where data are unavailable via CDAWeb. Hinode is a Japanese mission developed and launched by ISAS/JAXA, collaborating with NAOJ as a domestic partner,
NASA, and STFC (UK) as international partners. Scientific operation of the Hinode mission is conducted by the Hinode science team organized at ISAS/JAXA. This team mainly consists of scientists from institutes in the partner countries. Support for the post-launch operation is provided by JAXA and NAOJ (Japan), STFC (UK), NASA (USA), ESA, and NSC (Norway). The research leading to these results has received funding from the European Union's Seventh Programme for Research, Technological Development and Demonstration under Grant Agreement No. 284461 (eHEROES project). We gratefully acknowledge support from Marc DeRosa concerning PFSS modeling. L.v.D.G. acknowledges the Hungarian government for grant OTKA K-109276. The authors acknowledge STFC support under Consolidated Grant ST/H00260/1.

\section{REFERENCES}

Aellig, M. R., Hefti, S., Grunwaldt, H., et al. 1999, JGR, 104, 24769 Antiochos, S. K., Mikić, Z., Titov, V. S., et al. 2011, ApJ, 731, 112 Baker, D., van Driel-Gesztelyi, L., Mandrini, C. H., et al. 2009, ApJ, 705, 926 Banerjee, D., Teriaca, L., Gupta, G. R., et al. 2009, A\&A, 499, L29 Bemporad, A., \& Abbo, L. 2012, ApJ, 751, 110

Borrini, G., Gosling, J. T., Bame, S. J., \& Feldman, W. D. 1983, SoPh, 83, 367 Bradshaw, S. J., Aulanier, G., \& Del Zanna, G. 2011, ApJ, 743, 66 Brooks, D., \& Warren, H. P. 2012, ApJL, 760, L5

Brooks, D. H., Ugarte-Urra, I., \& Warren, H. P. 2015, NatCo, 6, 5947

Crooker, N. U., Kahler, S. W., Larson, D. E., \& Lin, R. P. 2004, JGR, 109, A03108

Culhane, J. L., Brooks, D. H., van Driel-Gesztelyi, L., et al. 2014, SoPh, 289, 3799

Culhane, J. L., Harra, L. K., James, A. M., et al. 2007, SoPh, 243, 19

Del Zanna, G. 2008, A\&A, 481, L69

Del Zanna, G., Aulanier, G., Klein, K., \& Török, T. 2011, A\&A, 526, A137

Doschek, G. A., Warren, H. P., Mariska, J. T., et al. 2008, ApJ, 686, 1362

Feldman, U., Widing, K. G., \& Warren, H. P. 1999, ApJ, 522, 1133

Fisk, L. A. 2003, JGR, 108, 1157

Gloeckler, G., Zurbuchen, T. H., \& Geiss, J. 2003, JGR, 108, 1158

Golub, L., DeLuca, E., Austin, G., et al. 2007, SoPh, 243, 63

Hara, H., Watanabe, T., Harra, L. K., et al. 2008, ApJL, 678, L67

Harra, L. K., Sakao, T., Mandrini, C. H., et al. 2008, ApJL, 676, L147

Hassler, D. M., Dammasch, I. E., Lemaire, P., et al. 1999, Sci, 283, 810

He, J.-S., Marsch, E., Curdt, W., et al. 2010a, A\&A, 519, A49

He, J.-S., Marsch, E., Tu, C.-Y., Guo, L.-J., \& Tian, H. 2010b, A\&A, 516, A14 Kamio, S., Curdt, W., Teriaca, L., \& Innes, D. E. 2011, A\&A, 529, A21

Kasper, J. C., Stevens, M. L., Lazarus, A. J., et al. 2007, ApJ, 660, 901

Ko, Y.-K., Raymond, J. C., Zurbuchen, T. H., et al. 2006, ApJ, 646, 1275

Kojima, M., Fujiki, K., Ohmi, T., et al. 1999, JGR, 104, 16993

Kosugi, T., Matsuzaki, K., Sakao, T., et al. 2007, SoPh, 243, 3

Liewer, P. C., Neugebauer, M., \& Zurbuchen, T. 2004, SoPh, 223, 209

Luhmann, J., Lee, C. O., Yan, L., et al. 2009, SoPh, 256, 285

Luhmann, J., Li, Y., Arge, C. N., et al. 2002, JGR, 107, 1154

Madjarska, M. S., Huang, Z., Doyle, J. G., \& Subramanian, S. 2012, A\&A, 545, A67

Mandrini, C. H., Nuevo, F. A., Vasquez, A. M., et al. 2014, SoPh, 289, 4151 McIntosh, S. W., \& De Pontieu, B. 2009, ApJL, 706, L80

Narang, N., Arbacher, R. T., Tian, H., et al. 2016, SoPh, 291, 1129

Neugebauer, M., Liewer, P. C., Smith, E. J., et al. 2002, JGR, 107, 1488

Neugebauer, M., Forsyth, R. J., Galvin, A. B., et al. 1998, JGR, 103, 14587

Nolte, J. T., \& Roelof, E. C. 1973, SoPh, 33, 241

Parker, E. N. 1958, ApJ, 128, 664

Richardson, I. G., \& Cane, H. V. 1995, JGR, 100, 23397

Sakao, T., Kano, R., Narukage, N., et al. 2007, Sci, 318, 1585

Schatten, K. H., Wilcox, J. M., \& Ness, N. F. 1969, SoPh, 6, 442

Schrijver, C. J., \& DeRosa, M. L. 2003, SoPh, 212, 165

Scott, J. T., Martens, P. C. H., \& Tarr, L. 2013, ApJ, 765, 82

Slemzin, V., Harra, L., Urnov, A., et al. 2013, SoPh, 286, 157

van Driel-Gesztelyi, L., Culhane, J. L., Baker, D., et al. 2012, SoPh, 281, 237

Wang, Y.-M., Ko, Y.-K., \& Grappin, R. 2009, ApJ, 691, 760

Zangrilli, L., \& Poletto, G. 2012, A\&A, 545, A8

Zurbuchen, T. H., \& Richardson, I. G. 2006, SSRv, 123, 31 